\title{
Adaptive Fuzzy Output Feedback Control for Partial State Constrained Nonlinear Pure Feedback Systems
}

\author{
Liping Wang $\mathbb{D}^{1},{ }^{1}$ Weiwei Sun $\mathbb{D},,^{1,2}$ and You $W u\left(\mathbb{D}{ }^{1}\right.$ \\ ${ }^{1}$ Institute of Automation, Qufu Normal University, Qufu 273165, China \\ ${ }^{2}$ School of Engineering, Qufu Normal University, Rizhao 276826, China \\ Correspondence should be addressed to Weiwei Sun; wwsun@hotmail.com
}

Received 9 May 2018; Accepted 4 July 2018; Published 24 July 2018

Academic Editor: Weihai Zhang

Copyright (c) 2018 Liping Wang et al. This is an open access article distributed under the Creative Commons Attribution License, which permits unrestricted use, distribution, and reproduction in any medium, provided the original work is properly cited.

\begin{abstract}
The adaptive fuzzy output feedback control problem for a class of pure feedback systems with partial state constraints is addressed in this paper. The fuzzy state observers are designed to estimate the unmeasured state while the fuzzy logic systems are used to approximate the unknown nonlinear functions. The proposed adaptive fuzzy output feedback controller can guarantee that the partial state constraints are not violated, and all closed-loop signals remain bounded by use of Barrier Lyapunov Functions (BLFs). A numerical example is presented to illustrate the effectiveness of the results in this paper.
\end{abstract}

\section{Introduction}

During the last decades, control design of nonlinear systems has attracted increasing interests. All kinds of control techniques have been proposed for both theoretical analysis and practical applications [1-6]. Many practical systems are inherently nonlinear and subject to many forms of constraints such as saturation and physical stoppages. Violation of the constraints may degrade the control performance and even make the system unstable. Therefore, the constraints handling in control design has attracted considerable attention [6-15]. There exist various techniques to tackle the constraints for nonlinear systems like nonlinear reference governor [7], invariance control [9], nonlinear model predictive control [11], etc.

Backstepping methodology is more effective in synthesis of robust and adaptive nonlinear controllers for various systems with parametric or dynamic nonlinearities and uncertainties [16-22]. The work in [20] constructs an adaptive tracking controller by introducing an auxiliary integrator subsystem and using the improved backstepping method such that the closed-loop system has a unique solution that is globally bounded in probability. The concept of Barrier Lyapunov Function (BLF), which is developed via Control Lyapunov Function (CLF) [23] in backstepping design method, was first proposed in [24]. The characteristic of BLF is that it will approach infinity whenever its arguments approach some limits. Transgression of constraints can be prevented through keeping BLF bounded in the closed-loop system. BLF-based backstepping control has been applied to many constrained nonlinear systems control synthesis [25-29]. Therein, $[24,30,31]$ have solved the BLF-based control problem of strict feedback nonlinear systems. The work [27] investigates the output tracking control problem of constrained nonlinear switch systems. The work [29] deals with the problem of adaptive dynamic surface control of nonlinear systems with unknown dead zone in pure feedback form. And the problem with respect to full state constraints is solved in $[32,33]$.

As we all know, the adaptive fuzzy control has an automatic learning capability which can adjust the adaptive parameters to deal with the uncertainty. Using the approximation property, fuzzy logic systems (FLSs) have been employed to tackle unknown nonlinear systems [26, 28, 34-36]. A control for nonlinear sampled systems with the guaranteed suboptimal performance achieved robust tracking by using fuzzy disturbance observer approach [26]. The work [34] studied an adaptive fuzzy dynamic surface control for nonlinear systems with fuzzy dead zone, unmodeled dynamics, dynamical disturbances, and 
unknown control gain functions. And the unknown system functions are approximated by the Takagi-Sugeno-type fuzzy logic systems. The work [36] addressed the adaptive control problem for nonlinear pure feedback systems based on fuzzy backstepping approach. Moreover, [28] constructed the approximation-based adaptive fuzzy tracking controller for non-strict-feedback stochastic nonlinear time-delay systems. As for the adaptive fuzzy observer design, to the best of the authors' knowledge, the existing results consider only the influence of full state constraints, and there is no further discussion about partial state constraints. There is seldom adaptive control method subject to partial state constraints and unmeasured state.

In this paper, we present an adaptive fuzzy backstepping tracking controller for a class of pure feedback nonlinear systems subject to unmeasured states and unknown nonlinear function. Firstly, the fuzzy state observers are designed to estimate the unmeasured state while the fuzzy logic systems are used to approximate the unknown nonlinear functions. Secondly, the proposed adaptive fuzzy output feedback controller can guarantee that the partial state constraints are not exceeded, and all closed-loop signals remain bounded with the using of BLF while the adaptive law for the estimations on uncertain parameters is constructed. A new coordinate transform is introduced during the process. Finally, a numerical example is given to validate our results presented in this paper.

The paper is organized as follows. Section 2 presents the problem formulation and some preliminaries. The main results are proposed in Section 3. Section 4 illustrates the effectiveness of the results by a numerical example.

\section{Problem Statement and Preliminaries}

2.1. Systems Description. Consider the following nonlinear pure feedback systems:

$$
\begin{aligned}
& \dot{x}_{i}=x_{i+1}+f_{i}\left(\bar{x}_{i}, x_{i+1}\right), \\
& \dot{x}_{n}=u+f_{n}\left(\bar{x}_{n}, u\right), \\
& y=x_{1},
\end{aligned}
$$

where $\bar{x}_{i}=\left[x_{1}, x_{2}, \ldots, x_{i}\right]^{\mathrm{T}} \in \mathbb{R}^{i}, i=1,2, \ldots, n$, are the state vectors of the systems, $u$ and $y$ are the input and output, respectively. The partition of the full states is constrained, i.e., constrained states $x_{s}=\left[x_{1}, x_{2}, \ldots, x_{n_{s}}\right]^{\mathrm{T}}$ and free states $x_{r}=\left[x_{n_{s+1}}, x_{n_{s+2}}, \ldots, x_{n}\right]^{\mathrm{T}}$. And the number sequences, $\left\{1,2, \ldots, n_{s}\right\}$ and $\left\{n_{s+1}, n_{s+2}, \ldots, n\right\}$, are both ascending. The states $x_{i}(t), i=1, \ldots, n_{s}$ are required to remain in the set $\left|x_{i}\right|<k_{c_{i}}$ with $k_{c_{i}}$ being positive constant, $\forall t \geq 0$. The nonlinear functions $f_{i}\left(\bar{x}_{i}, x_{i+1}\right), i=1,2, \ldots, n-1$ and $f_{n}\left(\bar{x}_{n}, u\right)$ are unknown nonlinear smooth functions, supposing that only the output signal is measured and other states are unmeasurable.

This paper is concerned with the problem of adaptive fuzzy output feedback control for system (1). Because of the existing unknown nonlinear functions, the fuzzy logic systems are employed to approximate the unknown nonlinear functions, and the fuzzy state observers are designed to handle the unmeasurable states. The following lemmas and assumptions related to backstepping design are given which will be used in the further analysis in the sequel.

Lemma 1 (see [24]). For any positive constants $k_{b_{i}}$, let $\mathscr{Z}:=$ $\left\{z \in \mathbb{R}^{l}:\left|z_{i}\right|<k_{b_{i}}, i=1,2, \ldots, n\right\} \subset \mathbb{R}^{n}$ and $\mathcal{N}:=\mathbb{R}^{l} \times \mathscr{Z} \subset$ $\mathbb{R}^{n+l}$ be open sets. Consider the system

$$
\dot{\eta}=h(t, \eta)
$$

where $\eta:=[w, z]^{\mathrm{T}} \in \mathcal{N}$ is the state, and the function $h: \mathbb{R}_{+} \times$ $\mathcal{N} \longrightarrow \mathbb{R}^{n+l}$ is piecewise continuous in $t$ and locally Lipschitz in $\eta$, uniformly in $t$, on $\mathbb{R}_{+} \times \mathscr{N}$. Let $\mathscr{Z}_{i}:=\left\{z_{i} \in \mathbb{R}:\left|z_{i}\right|<\right.$ $\left.k_{b_{i}}\right\} \subset \mathbb{R}$. Suppose that there exist positive definite functions $U$ : $\mathbb{R}^{l} \longrightarrow \mathbb{R}_{+}$and $V_{i}: \mathscr{Z}_{i} \longrightarrow \mathbb{R}_{+}(i=1,2, \ldots, n)$, both of which are also continuously differentiable on $\mathbb{R}^{l}$ and $\mathscr{Z}_{i}$, respectively, such that

$$
V_{i}\left(z_{i}\right) \longrightarrow \infty \quad \text { as } z_{i} \longrightarrow \pm k_{b_{i}}
$$

Let $V(\eta):=\sum_{i=1}^{n} V_{i}\left(z_{i}\right)+U(w)$ and $z(0) \in \mathscr{Z}$. If the inequality

$$
\dot{V}=\frac{\partial V}{\partial \eta} h \leq-\rho V+c,
$$

with constants $\rho>0, c>0$, holds in the set $z \in \mathscr{Z}$, then $z(t) \in \mathscr{Z}, \forall t \in[0, \infty)$.

Lemma 2 (see [30]). For any positive constants $k_{b}$, positive integer $p$, and any $z \in \mathbb{R}$ satisfying $|z|<k_{b}$, one has

$$
\log \frac{k_{b}^{2 p}}{k_{b}^{2 p}-z^{2 p}} \leq \frac{z^{2 p}}{k_{b}^{2 p}-z^{2 p}} .
$$

Proof. We define

$$
\begin{aligned}
q & =\frac{z^{2 p}}{k_{b}^{2 p}-z^{2 p}}-\log \frac{k_{b}^{2 p}}{k_{b}^{2 p}-z^{2 p}} \\
& =\frac{z^{2 p}}{k_{b}^{2 p}-z^{2 p}}-\log \left(1-\frac{z^{2 p}}{k_{b}^{2 p}-z^{2 p}}\right) .
\end{aligned}
$$

As $|z|<k_{b}$, we have $|z|^{2 p}<k_{b}^{2 p}$. Then, we can get the inequalities $0 \leq z^{2 p} /\left(k_{b}^{2 p}-z^{2 p}\right)<k_{b}^{2 p} /\left(k_{b}^{2 p}-z^{2 p}\right)=$ $1-z^{2 p} /\left(k_{b}^{2 p}-z^{2 p}\right)$ and $0 \leq z^{2 p} /\left(k_{b}^{2 p}-z^{2 p}\right)<1 / 2$. Let $\varsigma=z^{2 p} /\left(k_{b}^{2 p}-z^{2 p}\right)$ and then $q=-\log (1-\varsigma)+\varsigma$. The derivative of $q$ is given as $\dot{q}=(2-\varsigma) /(1-\varsigma)>0$. It shows that $q$ is continuously increasing and the minimum of $q$ is $q_{\text {min }}=0$. Thus, we get $\log \left(k_{b}^{2 p} /\left(k_{b}^{2 p}-z^{2 p}\right)\right) \leq z^{2 p} /\left(k_{b}^{2 p}-z^{2 p}\right)$, and $\log \left(k_{b}^{2 p} /\left(k_{b}^{2 p}-z^{2 p}\right)\right)=z^{2 p} /\left(k_{b}^{2 p}-z^{2 p}\right)$ if and only if $z=0$.

Lemma 3 (see [37]). Let $f(x)$ be a continuous function defined on a compact set $\Omega$, and, for any constant $\varepsilon>0$, there exists fuzzy logic system (12) such as

$$
f(x)=\widehat{f}(x \mid \theta)+\varepsilon(x), \quad|\varepsilon(x)| \leq \varepsilon .
$$


Lemma 4 (see [31]). For any $\beta \in \mathbb{R}^{n}$ and $\vartheta>0$, the following inequality holds:

$$
0<|\beta|-\beta \tanh \left(\frac{\beta}{9}\right) \leq \mu \vartheta, \quad \mu=0.2785 .
$$

Assumption 5 (see [24]). For any $k_{c_{1}}>0$, there exist positive constants $A_{0}, Y_{1}, Y_{2}, \ldots, Y_{n}$ such that the desired trajectory $y_{d}(t)$ and its time derivatives satisfy $\left|y_{d}(t)\right| \leq A_{0}<k_{c_{1}}$ and $\left|y_{d}^{(i)}(t)\right|<Y_{i}, i=1,2, \ldots, n$, for all $t \geq 0$.

Assumption 6. There exist known constants $m_{i}>0$ such that

$$
\left|f_{i}\left(x_{1}\right)-f_{i}\left(x_{2}\right)\right| \leq m_{i}\left\|x_{1}-x_{2}\right\|, \quad i=1,2, \ldots, n .
$$

Rewrite (1) as

$$
\begin{aligned}
& \dot{x}_{i}=x_{i+1}+f_{i}\left(\hat{\bar{x}}_{i}, \widehat{x}_{i+1, f}\right)+\Delta f_{i}, \\
& \dot{x}_{n}=u+f_{n}\left(\hat{\bar{x}}_{n}, u_{f}\right)+\Delta f_{n}, \\
& y=x_{1},
\end{aligned}
$$

where $\Delta f_{i}=f_{i}\left(\bar{x}_{i}, x_{i+1}\right)-f_{i}\left(\hat{\bar{x}}_{i}, \widehat{x}_{i+1, f}\right), i=1,2, \ldots, n-$ $1, \Delta f_{n}=f_{n}\left(\bar{x}_{n}, u\right)-f_{n}\left(\hat{\bar{x}}_{n}, u_{f}\right) \cdot \hat{\bar{x}}_{i}$ are the estimates of $\bar{x}_{i}$, which will be obtained by the state observer designed later; $\widehat{x}_{i+1, f}$ and $u_{f}$ are the filtered signals for $\widehat{x}_{i+1}$ and $u$, respectively. There exist known constants $\tau_{i}, i=1,2, \ldots, n$, such that $\left|\widehat{x}_{i+1}-\widehat{x}_{i+1, f}\right| \leq \tau_{i+1}\left(\tau_{1}=0\right)$. The filtered signals are defined as follows:

$$
\begin{gathered}
\hat{x}_{i, f}=H_{L}(s) \hat{x}_{i}, \\
u_{f}=H_{L}(s) u,
\end{gathered}
$$

where $H_{L}(s)$ is a Butterworth low-pass filter (LPF) $[26,36,38]$ with the cutoff frequency $\omega_{c}=1 \mathrm{rad} / \mathrm{s}$ for different values of $n$.

Remark 7. The filtered signals $\widehat{x}_{i+1, f}$ and $u_{f}$ are employed to avoid the so-called algebraic loop problem existing in $[29,39]$ and to design the state observer and controller for nonlinear pure feedback systems.

Remark 8. Based on the statements in $[26,36,38]$, most actuators have low-pass property and the replacements $\widehat{x}_{i, f} \approx$ $\widehat{x}_{i}$ and $u_{f} \approx u$ are reasonable in the controller design. Therefore, assume that $\left|\widehat{x}_{i}-\widehat{x}_{i, f}\right| \leq \tau_{i}$ with $\tau_{i}$ being known constants.

Then (10) can be further rewritten into the following state space form:

$$
\begin{aligned}
\dot{x}= & A x+K y+\sum_{i=1}^{n-1} B_{i}\left(f_{i}\left(\hat{\bar{x}}_{i}, \hat{\bar{x}}_{i+1, f}\right)+\Delta f_{i}\right) \\
& +B_{n}\left(f_{n}\left(\hat{\bar{x}}_{n}, u_{f}\right)+\Delta f_{n}+u\right),
\end{aligned}
$$

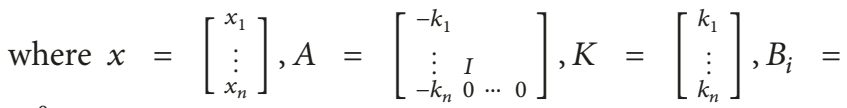
$\left[\begin{array}{c}0 \\ \vdots \\ 1 \\ \vdots \\ 0\end{array}\right], B_{n}=\left[\begin{array}{c}0 \\ \vdots \\ 1\end{array}\right]$. The vector $K$ is chosen to make matrix $A$ to be a strict Hurwitz matrix; i.e., for given a matrix $Q=Q^{T}>0$ there exists a matrix $P=P^{\mathrm{T}}>0$ satisfying

$$
A^{\mathrm{T}} P+P A=-2 Q \text {. }
$$

2.2. Design of FLSs and State Observer. To tackle the unknown nonlinear functions, the fuzzy logic systems are introduced as follows. then

Rule $j$ : if $x_{1}$ is $N_{j_{1}}$, and $x_{2}$ is $N_{j_{2}}$ and $\cdots$ and $x_{n}$ is $N_{j_{n}}$,

$$
y(x) \text { is } M_{j}, \quad j=1,2, \ldots, m,
$$

where $y$ is the output of the system, $N_{j_{1}}$ and $M_{j}$ denote fuzzy sets, and $m$ represents the number of fuzzy rules.

By using the singleton fuzzifier, center average defuzzification and product inference $[25,28]$, the final output can be expressed as follows:

$$
y(x)=\frac{\sum_{j=1}^{m} \tilde{y}_{j} \prod_{l=1}^{n} \mu_{N_{j_{1}}}\left(x_{l}\right)}{\sum_{j=1}^{m} \prod_{l=1}^{n} \mu_{N_{j_{1}}}\left(x_{l}\right)},
$$

where $\tilde{y}_{j}=\max _{y \in R} \mu_{M_{j}}(y) ; \mu_{N_{j_{1}}}\left(x_{l}\right)$ and $\mu_{M_{j}}(y)$ stand for membership functions with respect to fuzzy sets $N_{j_{1}}$ and $M_{j}$, respectively.

Define

$$
\varphi(x)=\frac{\prod_{l=1}^{n} \mu_{N_{j_{1}}}\left(x_{l}\right)}{\sum_{j=1}^{m} \prod_{l=1}^{n} \mu_{N_{j_{1}}}\left(x_{l}\right)}
$$

as the basis function vector. The ideal constant weight vector is $=\left[\tilde{y}_{1}, \tilde{y}_{2}, \ldots, \tilde{y}_{m}\right]^{\mathrm{T}}=\left[\theta_{1}, \theta_{2}, \ldots, \theta_{m}\right]^{\mathrm{T}}, \varphi(x)=$ $\left[\varphi_{1}(x), \varphi_{2}(x), \ldots, \varphi_{m}(x)\right]^{\mathrm{T}}$, and $\varphi_{i}(x)$ is chosen as Gaussian function; i.e., for $i=1,2, \ldots, m$,

$$
\varphi_{i}(x)=\exp \left[\frac{-\left(x-\mu_{i}\right)^{\mathrm{T}}\left(x-\mu_{i}\right)}{\eta_{i}^{2}}\right],
$$

where $\mu_{i}=\left[\mu_{i_{1}}, \mu_{i_{i}}, \ldots, \mu_{i_{n}}\right]^{\mathrm{T}}$ is the center vector, and $\eta_{i}$ is the width of the Gaussian function.

Then, the fuzzy output $y$ in (15) is described as

$$
y(x)=\theta^{\mathrm{T}} \varphi(x) .
$$

Accordingly, assume that the unknown nonlinear functions in (1) are approximated by the following fuzzy logic systems:

$$
\widehat{f}_{i}\left(\hat{\bar{x}}_{i}, \widehat{x}_{i+1, f} \mid \widehat{\theta}_{i}\right)=\widehat{\theta}_{i}^{\mathrm{T}} \varphi_{i}\left(\hat{\bar{x}}_{i}, \widehat{x}_{i+1, f}\right), \quad 1 \leq i \leq n,
$$

where $\widehat{x}_{n+1, f}=u_{f}$. The optimal weight vector $\theta_{i}^{*}$ is defined as

$$
\begin{aligned}
\theta_{i}^{*} & =\arg \min _{\theta_{i} \in \Omega_{i}}\left[\operatorname { s u p } _ { ( \overline { \overline { x } } _ { i } , \widehat { x } _ { i + 1 , f } ) \in U _ { i 1 } \times U _ { i 2 } } \left[\widehat{f}_{i}\left(\hat{\bar{x}}_{i}, \widehat{x}_{i+1, f} \mid \widehat{\theta}_{i}\right)\right.\right. \\
& \left.\left.-\widehat{f}_{i}\left(\hat{\bar{x}}_{i}, \widehat{x}_{i+1, f} \mid \theta_{i}^{*}\right)\right]\right]
\end{aligned}
$$


where $\Omega_{i}$ and $U_{i 1} \times U_{i 2}$ are compact regions for $\theta_{i}$ and $\left(\widehat{\bar{x}}_{i}, \widehat{x}_{i+1, f}\right)$, respectively.

Define

$$
f_{i}(x)=\theta_{i}^{* T} \varphi_{i}(x)+\varepsilon_{i}(x)=\widehat{\theta}_{i}^{T} \varphi_{i}(x)+\delta_{i}(x),
$$

where $\varepsilon_{i}^{*}$ and $\delta_{i}^{*}(i=1,2, \ldots, n)$ are known constants satisfying $\left|\varepsilon_{i}\right| \leq \varepsilon_{i}^{*}$ and $\left|\delta_{i}\right| \leq \delta_{i}^{*}$. Letting $w_{i}=\varepsilon_{i}-\delta_{i}, \quad i=$ $1,2, \ldots, n$, it is clear that there is an unknown constant $w_{i}^{*}>0$ such that $\left|w_{i}\right| \leq w_{i}^{*}=\varepsilon_{i}^{*}+\delta_{i}^{*}$.

Based on (12), design a fuzzy state observer as

$$
\begin{aligned}
\dot{\hat{x}}_{i} & =\widehat{x}_{i+1}+\widehat{f}_{i}\left(\hat{\bar{x}}_{i}, \widehat{x}_{i+1, f}\right)+k_{i}\left(y-\widehat{x}_{1}\right), \\
\dot{\hat{x}}_{n} & =u+\widehat{f}_{n}\left(\hat{\bar{x}}_{n}, u_{f}\right)+k_{n}\left(y-\widehat{x}_{1}\right), \\
y & =x_{1} .
\end{aligned}
$$

Let $e_{i}=x_{i}-\widehat{x}_{i}$ be an observer error vector. Then from (12) and (22), we have

$$
\dot{e}=A e+\delta+\triangle F,
$$

where $\delta=\left[\delta_{1}, \delta_{2}, \ldots, \delta_{n}\right]^{\mathrm{T}}$ and $\Delta F=\left[\Delta f_{1}, \Delta f_{2}, \ldots, \Delta f_{n}\right]^{\mathrm{T}}$. Substituting (23) into (22), we have

$$
\begin{aligned}
& \dot{e}_{i}=A e+\delta+\Delta F \\
& \dot{\hat{x}}_{i}=\widehat{x}_{i+1}+\widehat{f}_{i}\left(\widehat{\bar{x}}_{i}, \widehat{x}_{i+1, f}\right)+k_{i} e_{1}, \\
& \dot{\hat{x}}_{n}=u+\widehat{f}_{n}\left(\widehat{\bar{x}}_{n}, u_{f}\right)+k_{n} e_{1}, \\
& y=x_{1} .
\end{aligned}
$$

Consider the following Lyapunov function candidate:

$$
V_{0}=\frac{1}{2} e^{\mathrm{T}} P e
$$

Computing the time derivative of $V_{0}$, one has

$$
\dot{V}_{0}=e^{\mathrm{T}} P \dot{e}=e^{\mathrm{T}} P(A e+\delta+\Delta F) .
$$

According to Assumption 6, we get

$$
\begin{aligned}
\Delta f_{i} & =\left|f_{i}\left(\bar{x}_{i}, x_{i+1}\right)-f_{i}\left(\hat{\bar{x}}_{i}, \widehat{x}_{i+1, f}\right)\right| \\
& \leq m_{i}\|e\|^{2}+m_{i}\left|\widehat{x}_{i+1}-\widehat{x}_{i+1, f}\right| \leq m_{i}\|e\|^{2}+m_{i} \tau_{i+1} \\
& =m_{i}\|e\|^{2}+\tau_{i+1}^{\prime},
\end{aligned}
$$

where $\tau_{i+1}^{\prime}=m_{i} \tau_{i+1}$. It is clear that

$$
\begin{aligned}
\|\Delta F\|^{2} \leq & n m^{2}\|e\|^{2}+\left(\sum_{i=2}^{n+1} \tau_{i}^{\prime}\right)^{2} \\
e^{\mathrm{T}} P \triangle F \leq & \eta\|P\|^{2}\|e\|^{2}+\frac{1}{4 \eta} n m^{2}\|e\|^{2}+\eta\|P\|^{2}\|e\|^{2} \\
& +\frac{1}{4 \eta}\left(\sum_{i=2}^{n+1} \tau_{i}^{\prime}\right)^{2} \\
\leq & 2 \eta\|P\|^{2}\|e\|^{2}+\frac{1}{4 \eta} n m^{2}\|e\|^{2} \\
& +\frac{1}{4 \eta}\left(\sum_{i=2}^{n+1} \tau_{i}^{\prime}\right)^{2} .
\end{aligned}
$$

Using Young's inequality and (28), we get

$$
\begin{aligned}
\dot{V}_{0} \leq & -\lambda_{\min }(Q)\|e\|^{2}+\xi\|P\|^{2}\|e\|^{2}+\frac{1}{4 \xi}\left\|\delta^{*}\right\|^{2} \\
& +2 \eta\|P\|^{2}\|e\|^{2}+\frac{1}{4 \eta} n m^{2}\|e\|^{2}+\frac{1}{4 \eta}\left(\sum_{i=2}^{n+1} \tau_{i}^{\prime}\right)^{2} .
\end{aligned}
$$

To simplify the notation, let $\dot{V}_{0} \triangleq-p_{0}\|e\|^{2}+E_{0}$, where $-p_{0}=$ $-\lambda_{\text {min }}(Q)+\xi\|P\|^{2}+2 \eta\|P\|^{2}+(1 / 4 \eta) n m^{2}, E_{0}=(1 / 4 \xi)\left\|\delta^{*}\right\|^{2}+$ $(1 / 4 \eta)\left(\sum_{i=2}^{n+1} \tau_{i}^{\prime}\right)^{2}$.

\section{Main Results}

In this section, we propose a generalised design to deal with partial state constraint and the adaptive fuzzy output feedback controller which based on the backstepping technique and fuzzy state observer will be proposed. To guarantee the system performance, the virtual control signals and adaptive laws are designed. A new design procedure is presented which may cover some results related to the full state constraint [37]. To ensure that $x_{i}$ remains in the constrained region, we give the feasibility conditions with respect to the design parameters and an initial state region, i.e., $x(0) \in \Omega_{x(0)}$, where $\Omega_{x(0)}:=\left\{x \in \mathbb{R}^{n}:-a_{i} \leq x_{i} \leq b_{i}, i=1,2, \ldots, n\right\}$ with $a_{i}<k_{c_{i}}$ and $b_{i}<k_{c_{i}}$.

Let the tracking error

$$
z_{1}=y-y_{d}
$$

and the variables

$$
z_{i}=\widehat{x}_{i}-\alpha_{i-1}, \quad i=2, \ldots, n,
$$

where $\alpha_{i-1}$ is a virtual controller to be designed in Step $i$. 
Define $K_{z_{i}}=z_{i} /\left(k_{b_{i}}^{2}-z_{i}^{2}\right), k_{b_{1}}=k_{c_{1}}-Y_{0}$ and $\Omega_{z_{i}}=\left\{\left|z_{i}\right|<\right.$ $\left.k_{b_{i}}, i=1,2, \ldots, n\right\}$. Consider the BLF candidate combined with quadratic Lyapunov function as

$$
\begin{aligned}
V_{n}= & V_{0}+\sum_{i=1}^{n_{s}} \frac{1}{2} \log \frac{k_{b_{i}}^{2}}{k_{b_{i}}^{2}-z_{i}^{2}}+\sum_{i=1}^{n} \frac{1}{2 \gamma_{i}} \widetilde{\theta}_{i}^{\mathrm{T}} \widetilde{\theta}_{i}+\frac{1}{2{\overline{\gamma_{1}}}_{1}} \widetilde{\varepsilon}_{1}^{2} \\
& +\sum_{i=2}^{n} \frac{1}{2 \bar{\gamma}_{i}} \widetilde{w}_{i}^{2}+U
\end{aligned}
$$

where $U=\sum_{j=n_{s}+1}^{n}(1 / 2) z_{j}^{2}, j=n_{s}+1, \ldots, n$, and $\log (*)$ stands for the natural logarithm of $* . \gamma_{1}, \gamma_{i}, \bar{\gamma}_{1}$, and $\bar{\gamma}_{i}$ are positive constants. It can be proved that $V_{n}$ is continuously differentiable and positive definite on $\Omega_{z_{i}}$.

The detailed design procedures are given below.

Step 1. According to (19), (21), (24), and (30), the derivative of $z_{1}$ is calculated as follows:

$$
\dot{z}_{1}=\dot{y}-\dot{y}_{d}=z_{2}+\alpha_{1}+\theta_{1}^{*} \varphi_{1}+\varepsilon_{1}(x)+\Delta F_{1}-\dot{y}_{d} .
$$

The Lyapunov function is defined as

$$
V_{1}=V_{0}+\frac{1}{2} \log \frac{k_{b_{1}}^{2}}{k_{b_{1}}^{2}-z_{1}^{2}}+\frac{1}{2 \gamma_{1}} \widetilde{\theta}_{1}^{\mathrm{T}} \widetilde{\theta}_{1}+\frac{1}{2 \bar{\gamma}_{1}} \widetilde{\varepsilon}_{1}^{2} .
$$

Then, substituting (33) into (34), one can have

$$
\begin{aligned}
\dot{V}_{1}= & \dot{V}_{0}+\frac{z_{1} \dot{z}_{1}}{k_{b_{1}}^{2}-z_{1}^{2}}-\frac{1}{\gamma_{1}} \widetilde{\theta}_{1}^{\mathrm{T}} \dot{\hat{\theta}}_{1}-\frac{1}{\bar{\gamma}_{1}} \widetilde{\varepsilon}_{1} \dot{\bar{\varepsilon}}_{1} \\
= & \dot{V}_{0}+K_{z_{1}}\left(z_{2}+\alpha_{1}+\theta_{1}^{* \mathrm{~T}} \varphi_{1}+\varepsilon_{1}(x)+\Delta F_{1}-\dot{y}_{d}\right) \\
& -\frac{1}{\gamma_{1}} \widetilde{\theta}_{1}^{\mathrm{T}} \dot{\hat{\theta}}_{1}-\frac{1}{\bar{\gamma}_{1}} \widetilde{\varepsilon}_{1} \dot{\bar{\varepsilon}}_{1} .
\end{aligned}
$$

Design a virtual controller $\alpha_{1}$, adaptive law $\dot{\hat{\theta}}_{1}$, and $\dot{\widehat{\varepsilon}}_{1}$ as

$$
\begin{aligned}
& \alpha_{1}=-\lambda_{1} z_{1}-\widehat{\theta}_{1}^{\mathrm{T}} \varphi_{1}+\dot{y}_{d}-\widehat{\varepsilon}_{1} \tanh \left(\frac{K_{z_{1}}}{k}\right)-\frac{1}{2} K_{z_{1}}, \\
& \dot{\vec{\theta}}_{1}=-\sigma_{1} \widehat{\theta}_{1}+\gamma_{1} K_{z_{1}} \varphi_{1}, \\
& \dot{\widehat{\varepsilon}}_{1}=-\bar{\sigma}_{1} \widehat{\varepsilon}_{1}+\bar{\gamma}_{1} K_{z_{1}} \tanh \left(\frac{K_{z_{1}}}{k}\right),
\end{aligned}
$$

where $\widehat{\theta}_{1}$ is the estimation of $\theta_{1}^{*}$ and $\widetilde{\theta}_{j}=\theta_{j}^{*}-\widehat{\theta}_{j}, \widetilde{\varepsilon}_{1}=$ $\varepsilon_{1}^{*}-\widehat{\varepsilon}_{1}, \sigma_{1}, \bar{\sigma}_{1}$, and $k$ are the positive constants to be designed, respectively. Substituting (36) into (35), it yields

$$
\begin{aligned}
\dot{V}_{1}= & \dot{V}_{0}+K_{z_{1}}\left(z_{2}+\alpha_{1}+\theta_{1}^{* T} \varphi_{1}+\varepsilon_{1}(x)+\Delta F_{1}-\dot{y}_{d}\right) \\
& -\frac{1}{\gamma_{1}} \widetilde{\theta}_{1}^{\mathrm{T}}\left(-\sigma_{1} \widehat{\theta}_{1}+\gamma_{1} K_{z_{1}} \varphi_{1}\right) \\
& -\frac{1}{\bar{\gamma}_{1}} \widetilde{\varepsilon}_{1}\left(-\bar{\sigma}_{1} \widehat{\varepsilon}_{1}+\bar{\gamma}_{1} K_{z_{1}} \tanh \left(\frac{K_{z_{1}}}{k}\right)\right) \\
= & \dot{V}_{0}+K_{z_{1}}\left(z_{2}-\lambda_{1} z_{1}-\frac{1}{2} K_{z_{1}}+\Delta F_{1}\right) \\
& +\varepsilon_{1}(x) K_{z_{1}}-\varepsilon_{1}^{*} K_{z_{1}} \tanh \left(\frac{K_{z_{1}}}{k}\right)+\frac{\sigma_{1}}{\gamma_{1}} \widetilde{\theta}_{1}^{\mathrm{T}} \widehat{\theta}_{1} \\
& +\frac{\bar{\sigma}_{1}}{\bar{\gamma}_{1}} \widetilde{\varepsilon}_{1} \widehat{\varepsilon}_{1} \\
\leq & \dot{V}_{0}+K_{z_{1}}\left(z_{2}-\lambda_{1} z_{1}-\frac{1}{2} K_{z_{1}}+\Delta F_{1}\right) \\
& +0.2785 k \varepsilon_{1}^{*}+\frac{\sigma_{1}}{\gamma_{1}} \widetilde{\theta}_{1}^{\mathrm{T}} \widehat{\theta}_{1}+\frac{\bar{\sigma}_{1}}{\bar{\gamma}_{1}} \widetilde{\varepsilon}_{1} \widehat{\varepsilon}_{1} .
\end{aligned}
$$

Step $i\left(i=2,3, \ldots, n_{s}\right)$. The derivative of $z_{i}$ is calculated as follows:

$$
\begin{aligned}
\dot{z}_{i} & =\dot{\bar{x}}_{i}-\dot{\alpha}_{i-1} \\
& =z_{i+1}+\alpha_{i}+\theta_{i}^{*} \varphi_{i}+w_{i}(x)+k_{i} e_{1}-\dot{\alpha}_{i-1} .
\end{aligned}
$$

Choose the following Lyapunov function candidates:

$$
V_{i}=V_{i-1}+\frac{1}{2} \log \frac{k_{b_{i}}^{2}}{k_{b_{i}}^{2}-z_{i}^{2}}+\frac{1}{2 \gamma_{i}} \widetilde{\theta}_{i}^{\mathrm{T}} \widetilde{\theta}_{i}+\frac{1}{2 \bar{\gamma}_{i}} \widetilde{w}_{i}^{2},
$$

where $\widetilde{w}_{i}=w_{i}^{*}-\widehat{w}_{i}$. Similar to (35), one can have

$$
\begin{aligned}
\dot{V}_{i}= & \dot{V}_{i-1}+\frac{z_{i} \dot{z}_{i}}{k_{b_{i}}^{2}-z_{i}^{2}}-\frac{1}{\gamma_{i}} \widetilde{\theta}_{i}^{\mathrm{T}} \dot{\hat{\theta}}_{i}-\frac{1}{\bar{\gamma}_{i}} \widetilde{w}_{i} \dot{\widehat{w}}_{i} \\
= & \dot{V}_{i-1}-\frac{1}{\gamma_{i}} \widetilde{\theta}_{i}^{\mathrm{T}} \dot{\hat{\theta}}_{i}-\frac{1}{\bar{\gamma}_{i}} \widetilde{w}_{i} \dot{\hat{w}}_{i} \\
& +K_{z_{i}}\left(z_{i+1}+\alpha_{i}+\theta_{i}^{*} \varphi_{i}+w_{i}(x)+k_{i} e_{1}-\dot{\alpha}_{i-1}\right) .
\end{aligned}
$$

The virtual controller $\alpha_{i}$, adaptive law $\dot{\hat{\theta}}_{i}$, and $\dot{\widehat{\varepsilon}}_{i}$ are designed as

$$
\begin{aligned}
\alpha_{i}= & -\lambda_{i} z_{i}-\widehat{\theta}_{i}^{\mathrm{T}} \varphi_{i}-k_{i} e_{1}+\dot{\alpha}_{i-1}-\widehat{w}_{i} \tanh \left(\frac{K_{z_{i}}}{k}\right) \\
& -\frac{K_{z_{i-1}} z_{i}}{K_{z_{i}}} \\
\dot{\hat{\theta}}_{i}= & -\sigma_{i} \widehat{\theta}_{i}+\gamma_{i} K_{z_{i}} \varphi_{i}, \\
\dot{\widehat{w}}_{i}= & -\bar{\sigma}_{i} \widehat{w}_{i}+\bar{\gamma}_{i} K_{z_{i}} \tanh \left(\frac{K_{z_{i}}}{k}\right),
\end{aligned}
$$


where $\sigma_{i}$ and $\bar{\sigma}_{i}$ are positive constants. Substituting (41) into (40), the following inequality can be obtained:

$$
\begin{aligned}
\dot{V}_{i}= & \dot{V}_{i-1}-\frac{1}{\bar{\gamma}_{i}} \widetilde{w}_{i}\left(-\bar{\sigma}_{i} \widehat{w}_{i}+\bar{\gamma}_{i} K_{z_{i}} \tanh \left(\frac{K_{z_{i}}}{k}\right)\right) \\
& +K_{z_{i}}\left(z_{i+1}+\alpha_{i}+\theta_{i}^{*} \varphi_{i}+w_{i}(x)+k_{i} e_{1}-\dot{\alpha}_{i-1}\right) \\
& -\frac{1}{\gamma_{i}} \widetilde{\theta}_{i}^{\mathrm{T}}\left(-\sigma_{i} \widehat{\theta}_{i}+\gamma_{i} K_{z_{i}} \varphi_{i}\right) \\
\leq & \dot{V}_{0}+K_{z_{i}} z_{i+1}-\sum_{r=1}^{i} \lambda_{r} K_{z_{r}} z_{r}+\frac{1}{2} \Delta F_{1}^{2}+0.2785 k \varepsilon_{1}^{*} \\
& +0.2785 k \sum_{r=2}^{i} w_{r}^{*}+\sum_{r=1}^{i} \frac{\sigma_{r}}{\gamma_{r}} \widetilde{\theta}_{r}^{\mathrm{T}} \widehat{\theta}_{r}+\frac{\bar{\sigma}_{1}}{\bar{\gamma}_{1}} \widetilde{\varepsilon}_{1} \widehat{\varepsilon}_{1} \\
& +\sum_{r=2}^{i} \bar{\sigma}_{r} \widetilde{\bar{\gamma}}_{r} \widehat{w}_{r} .
\end{aligned}
$$

Step $n_{s}+1$. The derivative of $z_{n_{s}+1}$ is calculated as follows:

$$
\begin{aligned}
\dot{z}_{n_{s}+1}= & \dot{\hat{x}}_{n_{s}+1}-\dot{\alpha}_{n_{s}} \\
= & z_{n_{s}+2}+\alpha_{n_{s}+1}+\theta_{n_{s}+1}^{*} \varphi_{n_{s}+1}+w_{n_{s}+1}(x) \\
& +k_{n_{s}+1} e_{1}-\dot{\alpha}_{n_{s}} .
\end{aligned}
$$

The Lyapunov function is chosen as

$$
\begin{aligned}
V_{n_{s}+1}= & V_{n_{s}}+\frac{1}{2} z_{n_{s}+1}^{2}+\frac{1}{2 \gamma_{n_{s}+1}} \widetilde{\theta}_{n_{s}+1}^{\mathrm{T}} \widetilde{\theta}_{n_{s}+1} \\
& +\frac{1}{2 \bar{\gamma}_{n_{s}+1}} \widetilde{w}_{n_{s}+1}^{2} .
\end{aligned}
$$

Then, we have

$$
\begin{aligned}
& \dot{V}_{n_{s}+1}=\dot{V}_{n_{s}}+z_{n_{s}+1} \dot{z}_{n_{s}+1}-\frac{1}{\gamma_{n_{s}+1}} \widetilde{\theta}_{n_{s}+1}^{\mathrm{T}} \dot{\hat{\theta}}_{n_{s}+1}-\frac{1}{\bar{\gamma}_{n_{s}+1}} \\
& \cdot \widetilde{w}_{n_{s}+1} \dot{\widehat{w}}_{n_{s}+1}=\dot{V}_{n_{s}}+z_{n_{s}+1}\left(z_{n_{s}+2}+\alpha_{n_{s}+1}\right. \\
& \left.+\theta_{n_{s}+1}^{*} \varphi_{n_{s}+1}+w_{n_{s}+1}(x)+k_{n_{s}+1} e_{1}-\dot{\alpha}_{n_{s}}\right)-\frac{1}{\gamma_{n_{s}+1}} \\
& . \widetilde{\theta}_{n_{s}+1}^{\mathrm{T}} \dot{\hat{\theta}}_{n_{s}+1}-\frac{1}{\bar{\gamma}_{n_{s}+1}} \widetilde{w}_{n_{s}+1} \dot{\widehat{w}}_{n_{s}+1} .
\end{aligned}
$$

Design a virtual controller $\alpha_{n_{s}+1}$, adaptive law $\dot{\hat{\theta}}_{n_{s}+1}$, and $\dot{\bar{\varepsilon}}_{n_{s}+1}$ as

$$
\begin{aligned}
\alpha_{n_{s}+1}= & -\lambda_{n_{s}+1} z_{n_{s}+1}-\widehat{\theta}_{n_{s}+1}^{\mathrm{T}} \varphi_{n_{s}+1}-k_{n_{s}+1} e_{1}+\dot{\alpha}_{n_{s}} \\
& -\widehat{w}_{n_{s}+1} \tanh \left(\frac{z_{n_{s}+1}}{k}\right)-K_{z_{n_{s}}}, \\
\dot{\hat{\theta}}_{n_{s}+1}= & -\sigma_{n_{s}+1} \widehat{\theta}_{n_{s}+1}+\gamma_{n_{s}+1} z_{n_{s}+1} \varphi_{n_{s}+1}, \\
\dot{\widehat{w}}_{n_{s}+1}= & -\bar{\sigma}_{n_{s}+1} \widehat{w}_{n_{s}+1}+\bar{\gamma}_{n_{s}+1} z_{n_{s}+1} \tanh \left(\frac{z_{n_{s}+1}}{k}\right) .
\end{aligned}
$$

Substituting (46) into (45), the following inequality is obtained:

$$
\begin{aligned}
& \dot{V}_{n_{s}+1}=\dot{V}_{n_{s}}+z_{n_{s}+1}\left(z_{n_{s}+2}+\alpha_{n_{s}+1}+\theta_{n_{s}+1}^{*} \varphi_{n_{s}+1}\right. \\
& \left.+w_{n_{s}+1}(x)+k_{n_{s}+1} e_{1}-\dot{\alpha}_{n_{s}}\right)-\frac{1}{\bar{\gamma}_{n_{s}+1}} \\
& \cdot \widetilde{w}_{n_{s}+1}\left(-\bar{\sigma}_{n_{s}+1} \widehat{w}_{n_{s}+1}+\bar{\gamma}_{n_{s}+1} z_{n_{s}+1} \tanh \left(\frac{z_{n_{s}+1}}{k}\right)\right) \\
& \quad-\frac{1}{\gamma_{n_{s}+1}} \widetilde{\theta}_{n_{s}+1}^{\mathrm{T}}\left(-\sigma_{n_{s}+1} \widehat{\theta}_{n_{s}+1}+\gamma_{n_{s}+1} z_{n_{s}+1} \varphi_{n_{s}+1}\right) \leq \dot{V}_{0} \\
& +z_{n_{s}+1} z_{n_{s}+2}-\sum_{r=1}^{n_{s}} \lambda_{r} K_{z_{r}} z_{r}-\lambda_{n_{s}+1} z_{n_{s}+1}^{2}+\frac{1}{2} \Delta F_{1}^{2} \\
& +0.2785 k \varepsilon_{1}^{*}+0.2785 k \sum_{r=2}^{n_{s}+1} w_{r}^{*}+\sum_{r=1}^{n_{s}+1} \frac{\sigma_{r}}{\gamma_{r}} \widetilde{\theta}_{r}^{\mathrm{T}} \widehat{\theta}_{r}+\frac{\bar{\sigma}_{1}}{\bar{\gamma}_{1}} \\
& +\widetilde{\varepsilon}_{1} \widehat{\varepsilon}_{1}+\sum_{r=2}^{n_{s}+1} \bar{\sigma}_{r} \widetilde{\bar{\gamma}}_{r} \widehat{w}_{r} .
\end{aligned}
$$

Step $j\left(j=n_{s}+2, \ldots, n-1\right)$. The derivative of $z_{j}$ is calculated as follows:

$$
\begin{aligned}
\dot{z}_{j} & =\dot{\hat{x}}_{j}-\dot{\alpha}_{j-1} \\
& =z_{j+1}+\alpha_{j}+\theta_{j}^{*} \varphi_{j}+w_{j}(x)+k_{j} e_{1}-\dot{\alpha}_{j-1} .
\end{aligned}
$$

The Lyapunov function is defined as

$$
V_{j}=V_{j-1}+\frac{1}{2} z_{j}^{2}+\frac{1}{2 \gamma_{j}} \widetilde{\theta}_{j}^{\mathrm{T}} \widetilde{\theta}_{j}+\frac{1}{2 \bar{\gamma}_{j}} \widetilde{w}_{j}^{2}
$$

Then, one can have

$$
\begin{aligned}
\dot{V}_{j}= & \dot{V}_{j-1}+z_{j} \dot{z}_{j}-\frac{1}{\gamma_{j}} \widetilde{\theta}_{j}^{\mathrm{T}} \dot{\hat{\theta}}_{j}-\frac{1}{\bar{\gamma}_{j}} \widetilde{w}_{j} \dot{\hat{w}}_{j} \\
= & \dot{V}_{j-1}-\frac{1}{\gamma_{j}} \widetilde{\theta}_{j}^{\mathrm{T}} \dot{\hat{\theta}}_{j}-\frac{1}{\bar{\gamma}_{j}} \widetilde{w}_{j} \dot{\hat{w}}_{j} \\
& +z_{j}\left(z_{j+1}+\alpha_{j}+\theta_{j}^{*} \varphi_{j}+w_{j}(x)+k_{j} e_{1}-\dot{\alpha}_{j-1}\right) .
\end{aligned}
$$

Design a virtual controller $\alpha_{s+1}$, adaptive law $\dot{\hat{\theta}}_{s+1}$, and $\dot{\widehat{\varepsilon}}_{s+1}$ as

$$
\begin{aligned}
\alpha_{j}= & -\lambda_{j} z_{j}-\widehat{\theta}_{j}^{\mathrm{T}} \varphi_{j}-k_{j} e_{1}+\dot{\alpha}_{j-1}-\widehat{w}_{j} \tanh \left(\frac{z_{j}}{k}\right) \\
& -z_{j-1}, \\
\dot{\hat{\theta}}_{j}= & -\sigma_{j} \widehat{\theta}_{j}+\gamma_{j} z_{j} \varphi_{j}, \\
\dot{\widehat{w}}_{j}= & -\bar{\sigma}_{j} \widehat{w}_{j}+\bar{\gamma}_{j} z_{j} \tanh \left(\frac{z_{n_{j}}}{k}\right) .
\end{aligned}
$$


Substituting (51) into (50), the following inequality can be obtained:

$$
\begin{aligned}
\dot{V}_{j}= & \dot{V}_{j-1}-\frac{1}{\gamma_{j}} \widetilde{\theta}_{j}^{\mathrm{T}}\left(-\sigma_{j} \widehat{\theta}_{j}+\gamma_{j} z_{j} \varphi_{j}\right) \\
& +z_{j}\left(z_{j+1}+\alpha_{j}+\theta_{j}^{*} \varphi_{j}+w_{j}(x)+k_{j} e_{1}-\dot{\alpha}_{j-1}\right) \\
& -\frac{1}{\bar{\gamma}_{j}} \widetilde{w}_{j}\left(-\bar{\sigma}_{j} \widehat{w}_{j}+\bar{\gamma}_{j} z_{j} \tanh \left(\frac{z_{j}}{k}\right)\right) \\
\leq & \dot{V}_{0}+z_{j} z_{j+1}-\sum_{r=1}^{n_{s}} \lambda_{r} K_{z_{r}} z_{r}-\sum_{r=n_{s}+1}^{j} \lambda_{r} z_{r}^{2}+\frac{1}{2} \Delta F_{1}^{2} \\
& +0.2785 k \varepsilon_{1}^{*}+0.2785 k \sum_{r=2}^{j} w_{r}^{*}+\sum_{r=1}^{j} \frac{\sigma_{r}}{\gamma_{r}} \widetilde{\theta}_{r}^{\mathrm{T}} \widehat{\theta}_{r} \\
& +\frac{\bar{\sigma}_{1}}{\bar{\gamma}_{1}} \widetilde{\varepsilon}_{1} \widehat{\varepsilon}_{1}+\sum_{r=2}^{j} \bar{\gamma}_{r}{\widetilde{w_{r}}}_{r} \widehat{w}_{r} .
\end{aligned}
$$

Step $n$. The derivative of $z_{n}$ is calculated as follows:

$$
\dot{z}_{n}=\dot{\hat{x}}_{n}-\dot{\alpha}_{n-1}=u+\theta_{n}^{*} \varphi_{n}+w_{n}(x)+k_{n} e_{1}-\dot{\alpha}_{n-1} .
$$

Define the Lyapunov function as

$$
V_{n}=V_{n-1}+\frac{1}{2} z_{n}^{2}+\frac{1}{2 \gamma_{n}} \widetilde{\theta}_{n}^{\mathrm{T}} \widetilde{\theta}_{n}+\frac{1}{2 \bar{\gamma}_{n}} \widetilde{w}_{n}^{2}
$$

Then, one can have

$$
\begin{aligned}
\dot{V}_{n}= & \dot{V}_{n-1}+z_{n} \dot{z}_{n}-\frac{1}{\gamma_{n}} \widetilde{\theta}_{n}^{\mathrm{T}} \dot{\hat{\theta}}_{n}-\frac{1}{\bar{\gamma}_{n}} \widetilde{w}_{n} \dot{\widehat{w}}_{n} \\
= & \dot{V}_{n-1}+z_{n}\left(u+\theta_{n}^{*} \varphi_{n}+w_{n}(x)+k_{n} e_{1}-\dot{\alpha}_{n-1}\right) \\
& -\frac{1}{\gamma_{n}} \widetilde{\theta}_{n}^{\mathrm{T}} \dot{\hat{\theta}}_{n}-\frac{1}{\bar{\gamma}_{n}} \widetilde{w}_{n} \dot{\widehat{w}}_{n} .
\end{aligned}
$$

We choose the control law and the adaptive laws as

$$
\begin{aligned}
u= & -\lambda_{n} z_{n}-\widehat{\theta}_{n}^{\mathrm{T}} \varphi_{n}-k_{n} e_{1}+\dot{\alpha}_{n-1}-\widehat{w}_{n} \tanh \left(\frac{z_{n}}{k}\right) \\
& -z_{n-1}, \\
\dot{\hat{\theta}}_{n}= & -\sigma_{n} \widehat{\theta}_{n}+\gamma_{n} z_{n} \varphi_{n}, \\
\dot{\widehat{w}}_{n}= & -\bar{\sigma}_{n} \widehat{w}_{n}+\bar{\gamma}_{n} z_{n} \tanh \left(\frac{z_{n}}{k}\right) .
\end{aligned}
$$

Substituting (56) into (55), the following equality can be obtained:

$$
\begin{aligned}
\dot{V}_{n}= & \dot{V}_{n-1}+z_{n}\left(u+\theta_{n}^{*} \varphi_{n}+w_{n}(x)+k_{n} e_{1}-\dot{\alpha}_{n-1}\right) \\
& -\frac{1}{\bar{\gamma}_{n}} \widetilde{w}_{n}\left(-\bar{\sigma}_{n} \widehat{w}_{n}+\bar{\gamma}_{n} z_{n} \tanh \left(\frac{z_{n}}{k}\right)\right) \\
& -\frac{1}{\gamma_{n}} \widetilde{\theta}_{n}^{\mathrm{T}}\left(-\sigma_{n} \widehat{\theta}_{n}+\gamma_{n} z_{n} \varphi_{n}\right) \\
\leq & \dot{V}_{0}-\sum_{r=1}^{n_{s}} \lambda_{r} K_{z_{r}} z_{r}-\sum_{r=n_{s}+1}^{n} \lambda_{r} z_{r}^{2}+\frac{1}{2} \Delta F_{1}^{2} \\
& +0.2785 k \varepsilon_{1}^{*}+0.2785 k \sum_{r=2}^{n} w_{r}^{*}+\sum_{r=1}^{n} \frac{\sigma_{r}}{\gamma_{r}} \widetilde{\theta}_{r}^{\mathrm{T}} \widehat{\theta}_{r} \\
& +\frac{\bar{\sigma}_{1}}{\bar{\gamma}_{1}} \widetilde{\varepsilon}_{1} \widehat{\varepsilon}_{1}+\sum_{r=2}^{n} \frac{\bar{\sigma}_{r}}{\bar{\gamma}_{r}} \widetilde{w}_{r} \widehat{w}_{r} .
\end{aligned}
$$

According to Young's inequality, we have

$$
\frac{\sigma_{i}}{\gamma_{i}} \widetilde{\theta}_{i}^{\mathrm{T}} \widehat{\theta}_{i}=\frac{\sigma_{i}}{\gamma_{i}} \widetilde{\theta}_{i}^{\mathrm{T}}\left(\theta_{i}^{*}-\widetilde{\theta}_{i}\right) \leq \frac{\sigma_{i}}{2 \gamma_{i}}\left(\theta_{i}^{* \mathrm{~T}} \theta_{i}^{*}-\widetilde{\theta}_{i}^{\mathrm{T}} \widetilde{\theta}_{i}\right) .
$$

Then, the derivative of $V_{n}$ is given by

$$
\begin{aligned}
\dot{V}_{n} \leq & \dot{V}_{0}-\sum_{r=1}^{n_{s}} \lambda_{r} K_{z_{r}} z_{r}-\sum_{r=n_{s}+1}^{n} \lambda_{r} z_{r}^{2}+\frac{1}{2} \Delta F_{1}^{2} \\
& +0.2785 k \varepsilon_{1}^{*}+0.2785 k \sum_{r=2}^{n} w_{r}^{*} \\
& +\sum_{r=1}^{n} \frac{\sigma_{r}}{2 \gamma_{r}}\left(\theta_{r}^{*^{\mathrm{T}}} \theta_{r}^{*}-\widetilde{\theta}_{r}^{\mathrm{T}} \widetilde{\theta}_{r}\right)+\frac{\bar{\sigma}_{1}}{2 \bar{\gamma}_{1}}\left(\varepsilon_{1}^{*^{\mathrm{T}}} \varepsilon_{1}^{*}-\widetilde{\varepsilon}_{1}^{\mathrm{T}} \widetilde{\varepsilon}_{1}\right) \\
& +\sum_{r=2}^{n} \frac{\bar{\sigma}_{r}}{2 \bar{\gamma}_{r}}\left(w_{r}^{*^{\mathrm{T}}} w_{r}^{*}-\widetilde{w}_{r}^{\mathrm{T}} \widetilde{w}_{r}\right) .
\end{aligned}
$$

Combined with the above analysis, we have come to the following conclusions.

Theorem 9. Consider system (1). Assumptions 5 and 6 hold on the sets $\Omega_{z_{i}}$. For the virtual controller $\alpha_{i}, i=1,2, \ldots, n-1$, in (36), (41), and (51) and the actual controller $u$ in (56) and the adaptive laws in (36), (41), (51), and (56), the following properties hold:

(i) The proposed adaptive control scheme can guarantee that the tracking error converges to a bounded compact set $\Omega_{D}=\left\{\left|z_{1}\right|<D\right\}$.

(ii) All the signals in the closed-loop systems are bounded.

(iii) The partial state constraints are not violated.

Proof. (i) From Lemma 1, (59) can be rearranged into the form

$$
\dot{V} \leq-\rho V+c,
$$

where $\rho=\min \left\{\left(4 \eta+(1 / \eta)\|P\|^{2}\right) / 8\left(\lambda_{\min }(Q)-(\xi+2 \eta)\|P\|^{2}-\right.\right.$ $\left.\left.(1 / 4 \eta) n m^{2}-(1 / 2) m_{1}^{2}\right), 1 / 2 \lambda_{i}, 1 / \sigma_{j}, 1 / \bar{\sigma}_{1}, 1 / \bar{\sigma}_{j}\right\}, c=E_{n}+$ 
$\sum_{j=1}^{n}\left(\sigma_{j} / \gamma_{j}\right) \theta_{j}^{* T} \theta_{j}^{*}+\left(\bar{\sigma}_{1} / \bar{\gamma}_{1}\right) \varepsilon_{1}^{* 2}+\sum_{j=2}^{n}\left(\bar{\sigma}_{j} / \bar{\gamma}_{j}\right) w_{j}^{* 2}, E_{n}=E_{0}+$ $(1 / 2) \tau_{2}^{\prime 2}+k^{\prime} \varepsilon_{1}^{*}+\sum_{j=1}^{n} k^{\prime} w_{j}^{* 2}$, and $k^{\prime}=0.2785 k$. Adding $e^{\rho t}$ to both sides of the above inequality and integrating it over $[0, t]$, it has

$$
\begin{aligned}
V(t) & \leq\left[V(0)-\frac{c}{\rho}\right] e^{-\rho t}+\frac{c}{\rho} \leq V(0)+\frac{c}{\rho} \\
& \triangleq V(0)+C .
\end{aligned}
$$

From the preceding inequality and $V(t)$, we can conclude that $\log \left(k_{b_{j}}^{2} /\left(k_{b_{j}}^{2}-z_{j}^{2}\right)\right), e_{i}, \widetilde{\theta}_{j}, \widetilde{\varepsilon}_{1}, \widetilde{w}_{j}$ and $z_{j}$ are bounded, and $K_{z_{i}}$ is bounded. Due to the boundedness of $\theta_{j}^{*}, \varepsilon_{1}^{*}, w_{j}^{*}$ and $\widetilde{\theta}_{j}=\theta_{j}^{*}-\widehat{\theta}_{j}, \widetilde{\varepsilon}_{1}=\varepsilon_{1}^{*}-\widehat{\varepsilon}_{1}, \widetilde{w}_{j}=w_{j}^{*}-\widehat{w}_{j}$, then $\widehat{\theta}_{j}, \widehat{\varepsilon}_{1}$, and $\widehat{w}_{j}$ are bounded. It is easy to see that the tracking error converges to a bounded compact set.

(ii) According to (61), we define $B=$ $\sqrt{2(V(0)+C) / \lambda_{\min }(P)}$. From the form of $V(t)$, we have $\left|e_{i}\right| \leq\|e\| \leq B$. Since $x_{1}=z_{1}+y_{d}(t)$ and $\left|y_{d}(t)\right| \leq Y_{0}$, it can be shown that $\left|x_{1}\right| \leq\left|z_{1}\right|+\left|y_{d}(t)\right|<k_{b_{1}}+Y_{0}$. Suppose $k_{c_{1}}=k_{b_{1}}+Y_{0}$, and then $\left|x_{1}\right|<k_{c_{1}}$. To show $\left|x_{2}\right|<k_{c_{2}}$, it needs to confirm that there exists a positive constant $\bar{\alpha}_{1}$ such that $\left|\alpha_{1}\right| \leq \bar{\alpha}_{1}$. The boundedness of $x_{1}, y_{d}, \dot{y}_{d}, \widehat{\theta}_{1}, \widehat{\varepsilon}_{1}, K_{z_{1}}$ can be guaranteed because $\alpha_{1}=\alpha_{1}\left(x_{1}, y_{d}, \dot{y}_{d}, \widehat{\theta}_{1}, \widehat{\varepsilon}_{1}, K_{z_{1}}\right)$. It is easy to know from the definition of $\alpha_{1}$ that the supremum of $\alpha_{1}$ exists. In view of $\left|z_{2}\right|<k_{b_{2}}$ and $\widehat{x}_{2}=z_{2}+\alpha_{1}$, it has $\left|\widehat{x}_{2}\right|<k_{b_{2}}+\bar{\alpha}_{1}$. Due to $x_{2}=\widehat{x}_{2}+e_{2}$, the inequality $\left|x_{2}\right| \leq\left|\widehat{x}_{2}\right|+\left|e_{2}\right|<k_{b_{2}}+\bar{\alpha}_{1}+B$ holds. Let $k_{c_{2}}=k_{b_{2}}+\bar{\alpha}_{1}+B$, and then $\left|x_{2}\right|<k_{c_{2}}$. Similarly, it can show that $\left|x_{i+1}\right|<k_{c_{i+1}}, i=2,3, \ldots, n_{s}-1$, after verifying $\left|\alpha_{i}\right| \leq \bar{\alpha}_{i}$. Since (41), (51), and $\left|\alpha_{i-1}\right| \leq \bar{\alpha}_{i-1}$, then the controller $u$ is bounded. From the above analysis, we conclude that all the signals of the closed-loop system $x, \alpha, u, z, \widehat{\theta}_{j}, \widehat{\varepsilon}_{1}, \widehat{w}_{j}$ are bounded.

(iii) From the construction of (61), we get

$$
\log \frac{k_{b_{1}}^{2}}{k_{b_{1}}^{2}-z_{1}^{2}} \leq 2[V(0)-C] e^{-\rho t}+2 C .
$$

Taking exponentials on both sides, one has

$$
\frac{k_{b_{1}}^{2}}{k_{b_{1}}^{2}-z_{1}^{2}} \leq e^{2[V(0)-C] e^{-\rho t}+2 C} \text {. }
$$

Define

$$
D=k_{b_{1}} \sqrt{1-e^{-2[V(0)-C] e^{-\rho t}-2 C}} .
$$

Because $k_{b_{1}}^{2}-z_{1}^{2}>0$, it is easy to get $\left|z_{1}\right| \leq D$. If $V(0)=C$ then $\left|z_{1}\right| \leq k_{b_{1}} \sqrt{1-e^{-2 C}}=D$ holds. If $V(0) \neq C$, it can be concluded that, given any $D>k_{b_{1}} \sqrt{1-e^{-2 C}}$, there exists $T$ such that, for any $t>T$, it has $\left|z_{1}\right| \leq D$. This implies that $\left|z_{1}\right| \leq k_{b_{1}} \sqrt{1-e^{-2 C}}$ as $t \longrightarrow \infty$. That means $z_{1}$ can be made arbitrarily small. From above analysis, we can get that $\left|x_{i+1}\right|<$ $k_{c_{i+1}}\left(i=2,3, \ldots, n_{s}-1\right),\left|\alpha_{i-1}\right| \leq \bar{\alpha}_{i-1}$, and the controller $u$ is bounded. The variables $z_{i}=\widehat{x}_{i}-\alpha_{i-1}$, so that $z_{i}(i=$ $1,2, \ldots, n)$ is bound, and the systems states are not violated. This completes the proof.

\section{Illustrative Example}

In this section, we give an example to show how to apply the results proposed in this paper to investigate the stabilization of nonlinear pure feedback systems subject to partial state constraints.

Let us consider the following nonlinear systems:

$$
\begin{aligned}
& \dot{x}_{1}=x_{1} \cos \left(x_{1}\right)+\left(2 x_{1}^{2}+0.6\right) x_{2}, \\
& \dot{x}_{2}=x_{1} x_{2}+u+0.4 \sin (u), \\
& y=x_{1},
\end{aligned}
$$

where the state constraints are $\left|x_{1}\right|<0.5$; the reference signal is given as $y_{d}=0.1 \sin (\pi t / 3)+0.1 \cos (t / 3)$ for the tracking problem. It is unnecessary to give precise knowledge of the initial state $x(0)$. In this simulation, the figures are specific cases. Let $x_{1}=0.05, x_{2}=0.2, \widehat{x}_{1}=0, \widehat{x}_{2}=0$. Fuzzy membership functions for the variables $\widehat{x}_{1}, \widehat{x}_{2}, \widehat{x}_{2 f}$, and $u_{f}$ are given as follows:

$$
\begin{aligned}
& \mu_{F_{1}^{\prime}}\left(\widehat{x}_{1}\right)=\exp \left[-\frac{\left(\widehat{x}_{1}-5+2 l\right)^{2}}{2}\right], \\
& \mu_{F_{2}^{\prime}}\left(\widehat{x}_{2}\right)=\exp \left[-\frac{\left(\widehat{x}_{2}-3+l\right)^{2}}{5}\right], \\
& \mu_{F_{3}^{\prime}}\left(\widehat{x}_{2 f}\right)=\exp \left[-\frac{\left(\widehat{x}_{2 f}-3+l\right)^{2}}{5}\right], \\
& \mu_{F_{4}^{\prime}}\left(u_{f}\right)=\exp \left[-\frac{\left(u_{f}-5+3 l\right)^{2}}{7}\right],
\end{aligned}
$$

From [35], the fuzzy logic systems can be represented as

$$
\begin{aligned}
\widehat{f}_{1}\left(\widehat{x}_{1}, \widehat{x}_{2 f} \mid \theta_{1}\right) & =\theta_{1}^{\mathrm{T}} \varphi\left(\widehat{x}_{1}, \widehat{x}_{2 f}\right), \\
\widehat{f}_{2}\left(\widehat{x}_{1}, \widehat{x}_{2}, u_{f} \mid \theta_{2}\right) & =\theta_{2}^{\mathrm{T}} \varphi\left(\widehat{x}_{1}, \widehat{x}_{2}, u_{f}\right) .
\end{aligned}
$$

Letting $k_{1}=5$ and $k_{2}=5$, then the state observer can be constructed as

$$
\begin{aligned}
& \dot{\hat{x}}_{1}=\widehat{x}_{2}+\widehat{f}_{1}\left(\widehat{x}_{1}, \widehat{x}_{2 f} \mid \theta_{1}\right)+5\left(y-\widehat{x}_{1}\right), \\
& \dot{\hat{x}}_{2}=u+\widehat{f}_{2}\left(\widehat{x}_{1}, \widehat{x}_{2}, u_{f} \mid \theta_{2}\right)+5\left(y-\widehat{x}_{1}\right) .
\end{aligned}
$$


The virtual control function $\alpha_{1}$ and controller $u$ as well as the adaptive laws $\widehat{\theta}_{1}, \widehat{\theta}_{2}, \widehat{\varepsilon}_{1}$ and $\widehat{w}_{2}$ are as follows:

$$
\begin{aligned}
\alpha_{1}= & -\lambda_{1} z_{1}-\widehat{\theta}_{1}^{\mathrm{T}} \varphi_{1}+\dot{y}_{d}-\widehat{\varepsilon}_{1} \tanh \left(\frac{K_{z_{1}}}{k}\right)-\frac{1}{2} K_{z_{1}}, \\
u= & -\lambda_{2} z_{2}-\widehat{\theta}_{2}^{\mathrm{T}} \varphi_{2}-k_{2} e_{1}+\dot{\alpha}_{1}-\widehat{w}_{2} \tanh \left(\frac{z_{2}}{k}\right) \\
& -z_{1}, \\
\dot{\hat{\theta}}_{1}= & -\sigma_{1} \widehat{\theta}_{1}+\gamma_{1} K_{z_{1}} \varphi_{1}, \\
\dot{\widehat{\varepsilon}}_{1}= & -\bar{\sigma}_{1} \widehat{\varepsilon}_{1}+\bar{\gamma}_{1} K_{z_{1}} \tanh \left(\frac{K_{z_{1}}}{k}\right), \\
\dot{\hat{\theta}}_{2}= & -\sigma_{2} \widehat{\theta}_{2}+\gamma_{2} z_{2} \varphi_{2}, \\
\dot{\hat{w}}_{2}= & -\bar{\sigma}_{2} \widehat{w}_{2}+\bar{\gamma}_{2} z_{2} \tanh \left(\frac{z_{2}}{k}\right) .
\end{aligned}
$$

Set the design parameters in the above control scheme as

$$
\begin{aligned}
& \lambda_{1}=1, \\
& \lambda_{2}=2, \\
& \gamma_{1}=1, \\
& \gamma_{2}=1, \\
& \bar{\gamma}_{1}=1, \\
& \bar{\gamma}_{2}=1 ; \\
& \sigma_{1}=1, \\
& \sigma_{2}=1, \\
& \bar{\sigma}_{1}=1.5, \\
& \bar{\sigma}_{2}=0.5, \\
& k=0.2 .
\end{aligned}
$$

Let $k_{b_{1}}=0.2$, and from calculation we know it is valid. Choose the initial conditions of adaptive parameters as $\widehat{\theta}_{1}=$ $(1,1,1,1,1), \widehat{\varepsilon}_{1}=1, \widehat{\theta}_{2}=(1,1,1,1,1), \widehat{w}_{2}=1$.

The simulation is given in Figures 1-8. Figure 1 shows the trajectory of the state $x$. Figure 2 is the swing curve of the control signal $u$. Figure 3 is the state $x_{1}$ which remains in the constraint region; it shows the trajectory of the state $x$. Figure 4 stands for the variables of $z_{1}$ and $z_{2}$, and these error variables can not violate their bounds. Figures 5 and 6 are used to illustrate the trajectories of the system states $x_{1}, x_{2}$ and the observer states $\widehat{x}_{1}, \hat{x}_{2}$. Figures 7 and 8 show that all the signals in the closed-loop system are bounded. It is clear that the nonlinear pure feedback system subject to partial constraints under the output feedback law is bounded. From the simulation, we can conclude that the results proposed in Theorem 9 are very practicable in stability of nonlinear pure feedback systems with partial constraints. Meanwhile, it is a good tool in analyzing the stability problems of some

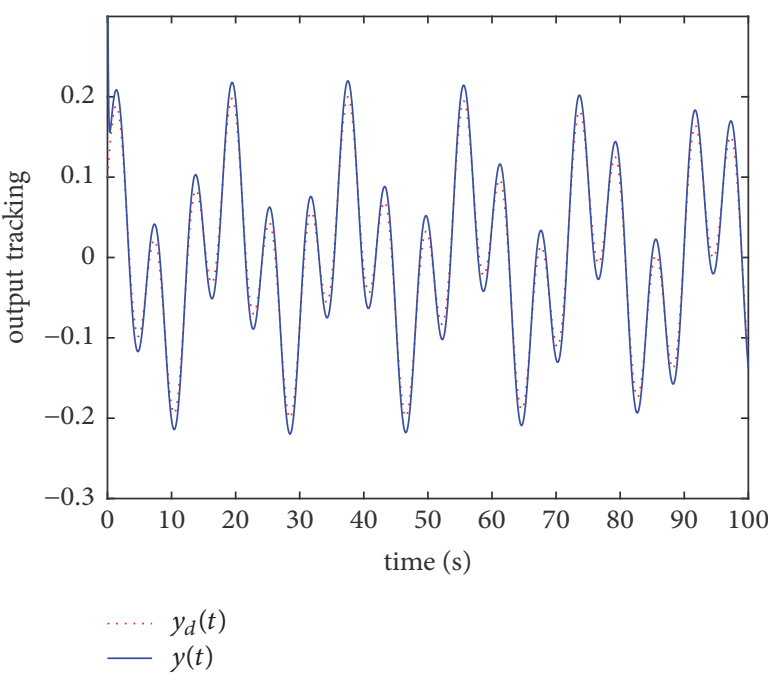

FIGURE 1: The tracking result.

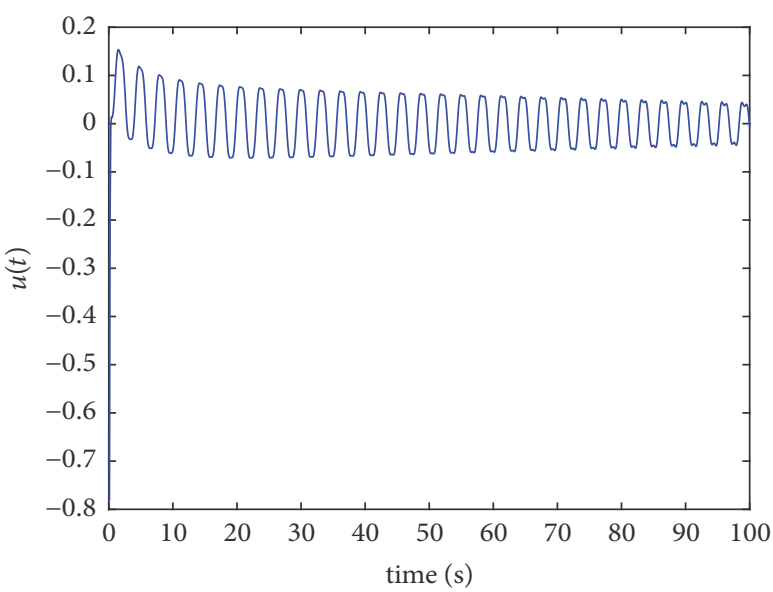

Figure 2: The control $u$.

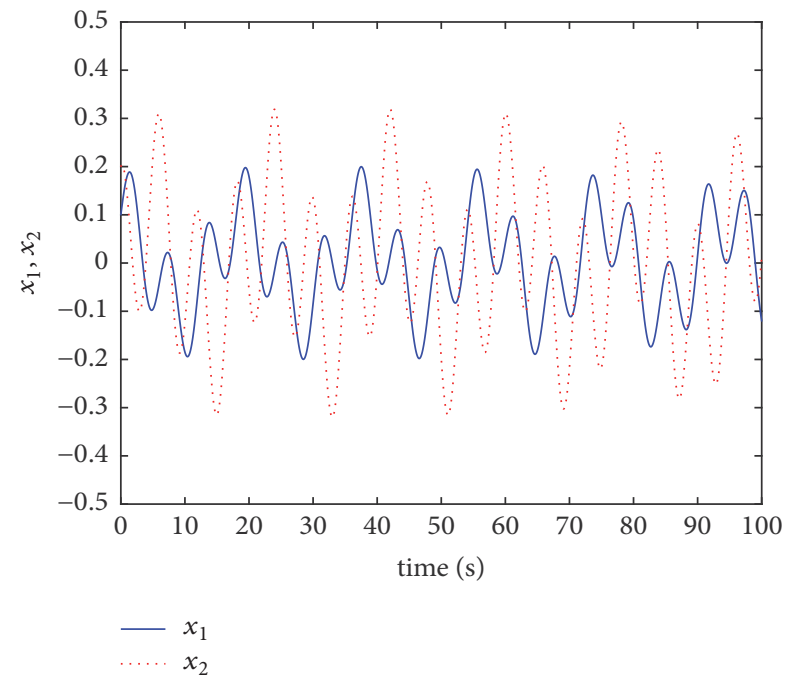

FIgURE 3: States $x_{1}$ and $x_{2}$. 


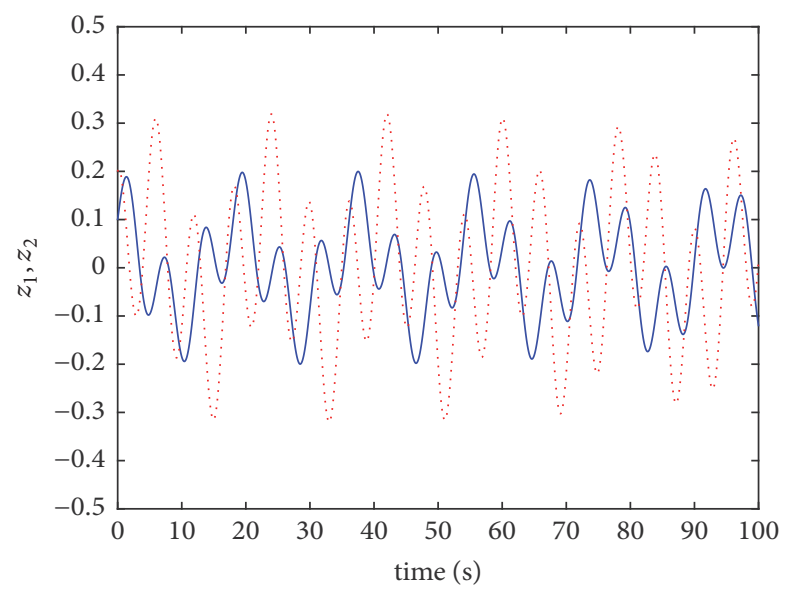

$$
z_{1}
$$

Figure 4: Variables $z_{1}$ and $z_{2}$.

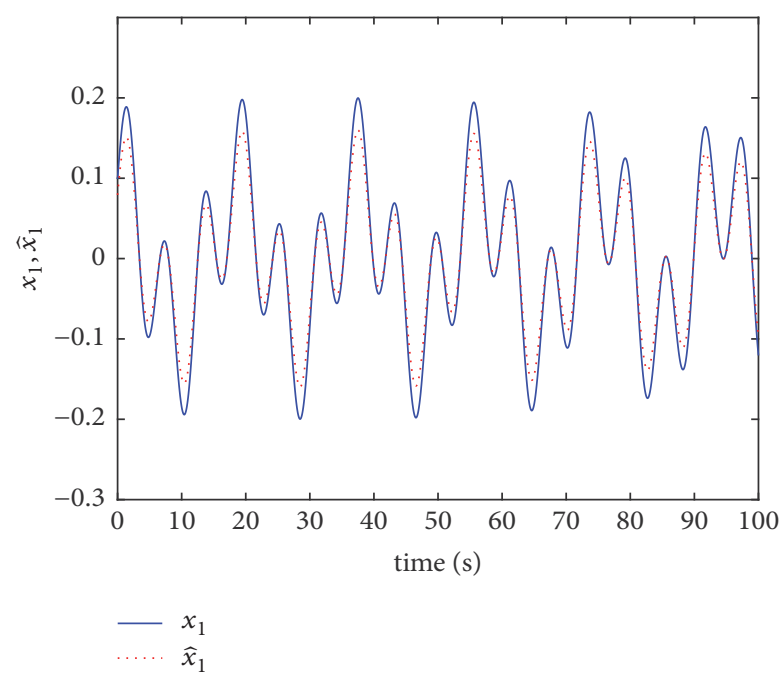

Figure 5: States $x_{1}$ and $\hat{x}_{1}$.

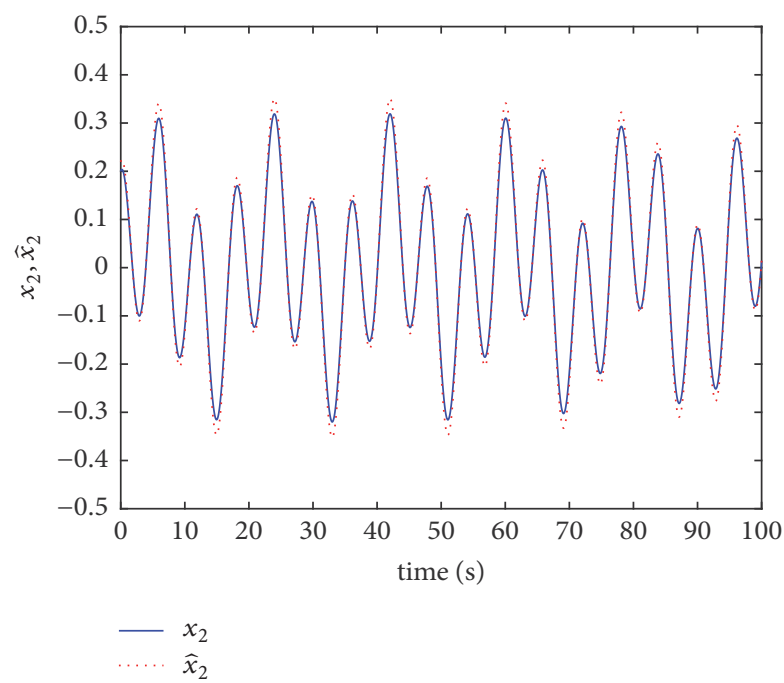

Figure 6: States $x_{2}$ and $\widehat{x}_{2}$.

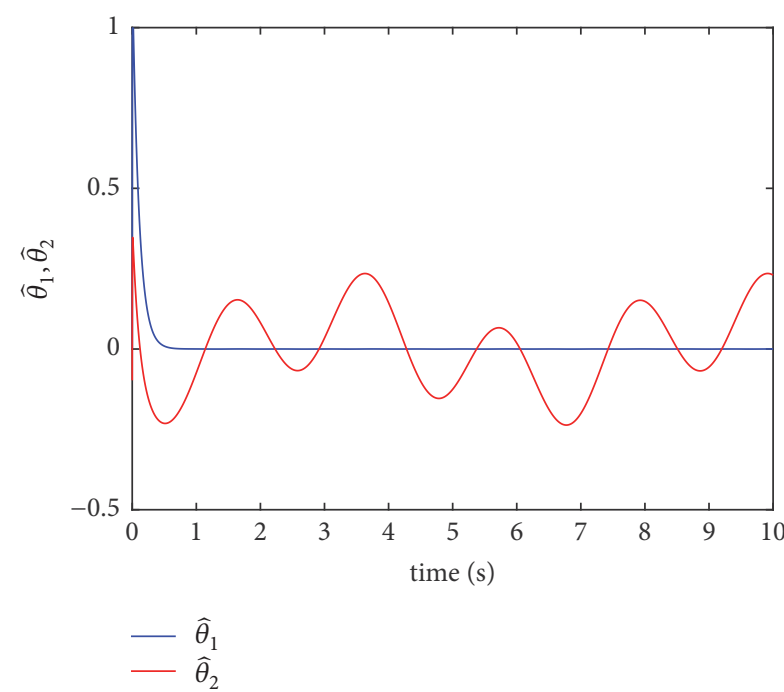

FIGURE 7: Parameter estimates $\widehat{\theta}_{1}, \widehat{\theta}_{2}$.

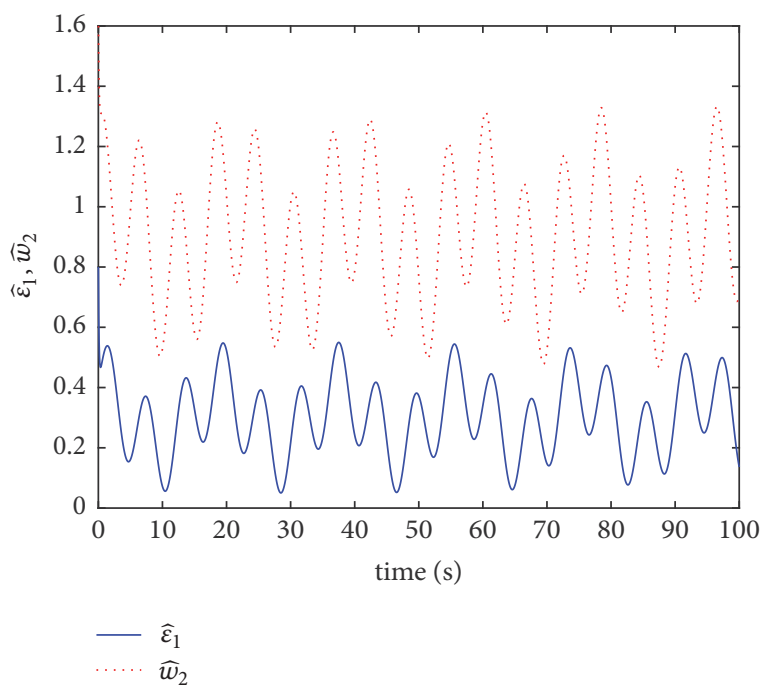

FIGURE 8: Parameter estimates $\widehat{\varepsilon}_{1}, \widehat{w}_{2}$.

classes of nonlinear pure feedback systems in the presence of constraint.

\section{Conclusions}

In this paper, the tracking control problem of a class of nonlinear pure feedback systems subject to partial state constraints and adaptive fuzzy output feedback controls has been investigated by use of BLFs and backstepping. The output feedback control law, by which the stability of the closedloop system is guaranteed, is determined by constraints. Simulations show that the results obtained in this paper are very practicable in analyzing the stability of some classes of nonlinear pure feedback systems. 


\section{Data Availability}

No data were used to support this study.

\section{Conflicts of Interest}

The authors declare that they have no conflicts of interest.

\section{Acknowledgments}

This work was supported by the National Natural Science Foundation of China (no. 61703232) and the Natural Science Foundation of Shandong Province (nos. ZR2017MF068, ZR2017QF013).

\section{References}

[1] L. Guo, L. Liu, and Y. Wu, "Existence of positive solutions for singular fractional differential equations with infinite-point boundary conditions," Nonlinear Analysis: Modelling and Control, vol. 21, no. 5, pp. 635-650, 2016.

[2] F. Li and Y. Bao, "Uniform stability of the solution for a memorytype elasticity system with nonhomogeneous boundary control condition," Journal of Dynamical and Control Systems, vol. 23, no. 2, pp. 301-315, 2017.

[3] S. Rahmani, N. Mendalek, and K. Al-Haddad, "Experimental design of a nonlinear control technique for three-phase shunt active power filter," IEEE Transactions on Industrial Electronics, vol. 57, no. 10, pp. 3364-3375, 2010.

[4] W. Sun and L. Peng, "Observer-based robust adaptive control for uncertain stochastic Hamiltonian systems with state and input delays," Nonlinear Analysis: Modelling and Control, vol. 19, no. 4, pp. 626-645, 2014.

[5] W. Sun and B. Fu, "Adapative control of time-varying uncertain non-linear systems with input delay: a Hamiltonian approach," IET Control Theory and Applications, vol. 10, no. 15, pp. 18441858, 2016.

[6] W. Sun, K. Wang, C. Nie et al., "Energy-based controller design of stochasic magnetic levitation system," Mathematical Problems in Engineering, vol. 2017, 6 pages, 2017.

[7] A. Bemporad, "Reference governor for constrained nonlinear systems," Institute of Electrical and Electronics Engineers Transactions on Automatic Control, vol. 43, no. 3, pp. 415-419, 1998.

[8] G. Chen, J. Xia, G. Zhuang, and J. Zhao, "Improved delaydependent stabilization for a class of networked control systems with nonlinear perturbations and two delay components," Applied Mathematics and Computation, vol. 316, pp. 1-17, 2018.

[9] T. Hu and Z. Lin, Control Systems with Actuator Saturation: Analysis and Design, Birkhäuser, Boston, Mass, USA, 2001.

[10] H. Li and Y. Wang, "Controllability analysis and control design for switched Boolean networks with state and input constraints," SIAM Journal on Control and Optimization, vol. 53, no. 5, pp. 2955-2979, 2015.

[11] D. Q. Mayne, J. B. Rawlings, C. V. Rao, and P. O. M. Scokaert, "Constrained model predictive control: stability and optimality," Automatica, vol. 36, no. 6, pp. 789-814, 2000.

[12] W. Sun, "Stabilization analysis of time-delay Hamiltonian systems in the presence of saturation," Applied Mathematics and Computation, vol. 217, no. 23, pp. 9625-9634, 2011.
[13] C. Wang, Y. Wu, and J. Yu, "Barrier Lyapunov functions-based adaptive control for nonlinear purefeedback systems with timevarying full state constraints," International Journal of Control, Automation and Systems, vol. 15, no. 6, pp. 2714-2722, 2017.

[14] C. Wang, Y. Wu, and J. Yu, "Barrier Lyapunov functions-based dynamic surface control for pure-feedback systems with full state constraints," IET Control Theory and Applications, vol. 11, no. 4, pp. 524-530, 2017.

[15] J. Xia, G. Chen, and W. Sun, "Extended dissipative analysis of generalized Markovian switching neural networks with two delay components," Neurocomputing, vol. 260, pp. 275-283, 2017.

[16] Z.-P. Jiang, "A combined backstepping and small-gain approach to adaptive output feedback control," Automatica, vol. 35, no. 6, pp. 1131-1139, 1999.

[17] M. Krstic, I. Kanellakopoulos, and P. Kokotovic, Nonlinear and Adaptive Control Design, Wiley, New York, NY, USA, 1995.

[18] B. Ren, S. S. Ge, K. P. Tee, and T. H. Lee, "Adaptive neural control for output feedback nonlinear systems using a barrier lyapunov function," IEEE Transactions on Neural Networks and Learning Systems, vol. 21, no. 8, pp. 1339-1345, 2010.

[19] X. Xie and M. Jiang, "Output feedback stabilization of stochastic feedforward nonlinear time-delay systems with unknown output function," International Journal of Robust and Nonlinear Control, vol. 28, no. 1, pp. 266-280, 2018.

[20] L. Yao and W. Zhang, "Adaptive tracking control for a class of random pure-feedback nonlinear systems with Markovian switching," International Journal of Robust and Nonlinear Control, vol. 28, no. 8, pp. 3112-3126, 2018.

[21] Z. Q. Zhang, S. Y. Xu, and B. Y. Zhang, "Exact tracking control of nonlinear systems with time delays and dead-zone input," Automatica, vol. 52, pp. 272-276, 2015.

[22] Z. Zhang, S. Xu, and B. Zhang, "Asymptotic tracking control of uncertain nonlinear systems with unknown actuator nonlinearity," IEEE Transactions on Automatic Control, vol. 59, no. 5, pp. 1336-1341, 2014.

[23] K. B. Ngo, R. Mahony, and Z. Jiang, "Integrator backstepping using barrier functions for systems with multiple state constraints," in Proceedings of the 44th IEEE Conference On Decision and Control, and the European Control Conference 2005, pp. 1215, Seville, Spain, 2005.

[24] K. P. Tee, S. S. Ge, and E. H. Tay, "Barrier Lyapunov functions for the control of output-constrained nonlinear systems," Automatica, vol. 45, no. 4, pp. 918-927, 2009.

[25] M. A. Khanesar, O. Kaynak, S. Yin, and H. Gao, "Adaptive indirect fuzzy sliding mode controller for networked control systems subject to time-varying network-induced time delay," IEEE Transactions on Fuzzy Systems, vol. 23, no. 1, pp. 205-214, 2015.

[26] E. Kim and C. W. Park, "Fuzzy disturbance observer approach to robust tracking control of nonlinear sampled systems with the guaranteed suboptimal performance," IEEE Transactions on Cybernetics, vol. 34, pp. 1574-1581, 2004.

[27] B. Niu and J. Zhao, "Barrier Lyapunov functions for the output tracking control of constrained nonlinear switched systems," Systems \& Control Letters, vol. 62, no. 10, pp. 963-971, 2013.

[28] H. Wang, X. Liu, K. Liu, and H. R. Karimi, "Approximationbased adaptive fuzzy tracking control for a class of nonstrictfeedback stochastic nonlinear time-delay systems," IEEE Transactions on Fuzzy Systems, vol. 23, no. 5, pp. 1746-1760, 2015. 
[29] T. P. Zhang and S. S. Ge, "Adaptive dynamic surface control of nonlinear systems with unknown dead zone in pure feedback form," Automatica, vol. 44, no. 7, pp. 1895-1903, 2008.

[30] K. P. Tee and S. S. Ge, "Control of nonlinear systems with partial state constraints using a barrier lyapunov function," International Journal of Control, vol. 84, no. 12, pp. 2008-2023, 2011.

[31] K. P. Tee, B. Ren, and S. S. Ge, "Control of nonlinear systems with time-varying output constraints," Automatica, vol. 47, no. 11, pp. 2511-2516, 2011.

[32] D. P. Li, D. J. Li, Y. J. Liu, S. Tong, and C. L. P. Chen, "Approximation-based adaptive neural tracking control of nonlinear mimo unknown time-varying delay systems with full state constraints," IEEE Transactions on Cybernetics, vol. 47, no. 10, pp. 3100-3109, 2017.

[33] T. Zhang, M. Xia, Y. Yi, and Q. Shen, "Adaptive Neural Dynamic Surface Control of Pure-Feedback Nonlinear Systems With Full State Constraints and Dynamic Uncertainties," IEEE Transactions on Systems, Man, and Cybernetics: Systems, vol. 47, no. 8, pp. 2378-2387, 2017.

[34] H. Su and W. Zhang, "A combined backstepping and dynamic surface control to adaptive fuzzy state-feedback control," International Journal of Adaptive Control and Signal Processing, vol. 31, no. 11, pp. 1666-1685, 2017.

[35] T. Wang, Adaptive Fuzzy Systems and Control, Prentice Hall, Englewood Cliffs, NJ, USA, 1994.

[36] A.-M. Zou, Z.-G. Hou, and M. Tan, "Adaptive control of a class of nonlinear pure-feedback systems using fuzzy backstepping approach," IEEE Transactions on Fuzzy Systems, vol. 16, no. 4, pp. 886-897, 2008.

[37] J. Yi, J. Li, J. Li, and J. Wu, "Adaptive fuzzy output feedback control for a class of nonlinear pure-feedback systems with full state constraints," in Proceedings of the 2017 36th Chinese Control Conference (CCC), pp. 862-867, Dalian, China, July 2017.

[38] Z. Yang, S. Hara, S. Kanae, and K. Wada, "Robust output feedback control of a class of nonlinear systems using a disturbance observer," IEEE Transactions on Control Systems Technology, vol. 19, no. 2, pp. 256-268, 2011.

[39] D. Wang and J. Huang, "Adaptive neural network control of a class of uncertain nonlinear systems in pure-feedback form," Automatica, vol. 42, no. 8, pp. 1365-1372, 2002. 


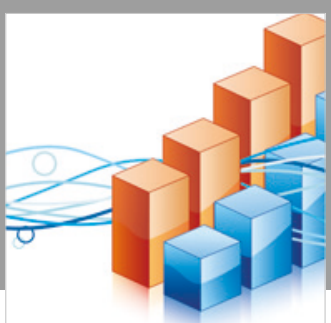

Advances in

Operations Research

\section{-n-m}
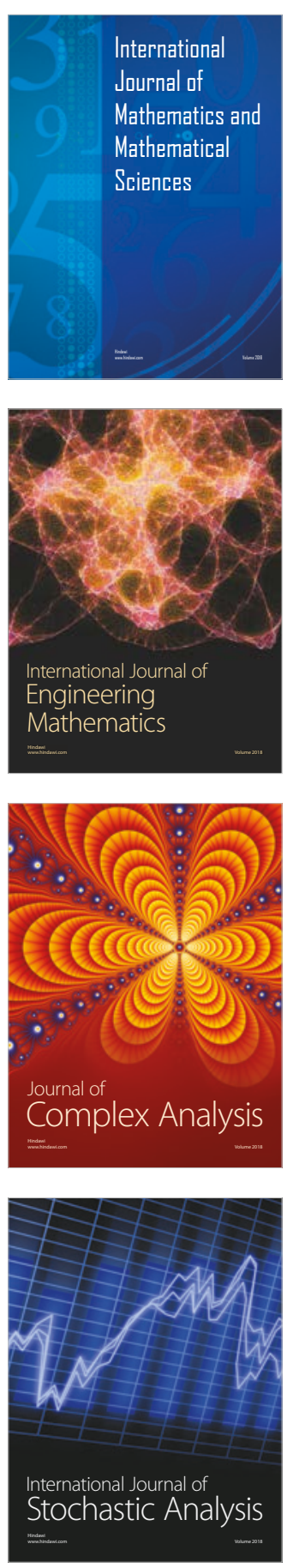
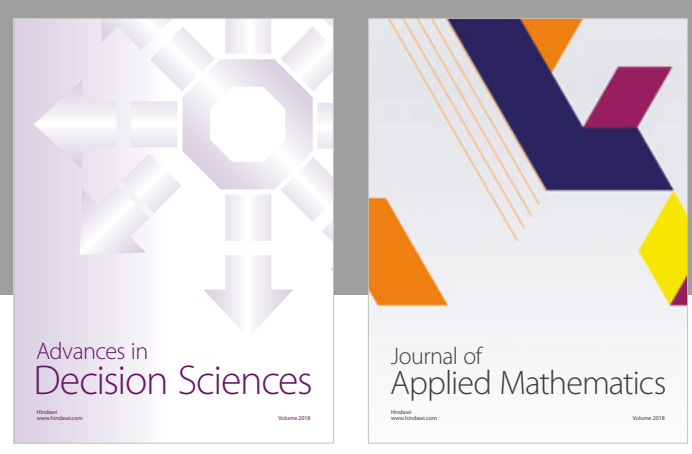

Journal of

Applied Mathematics
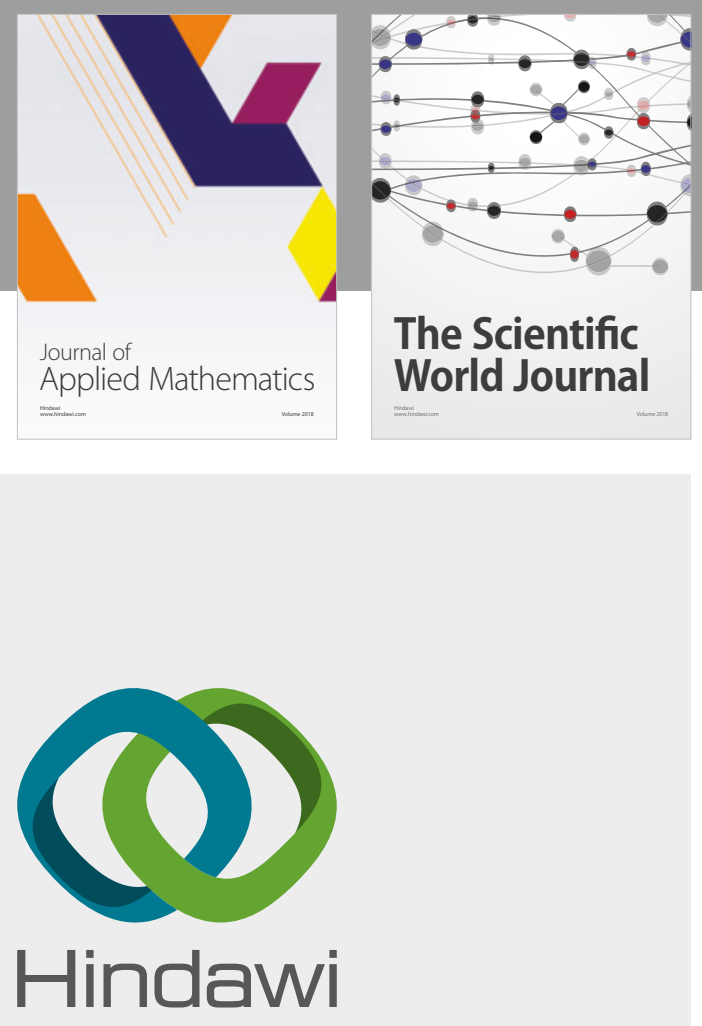

Submit your manuscripts at

www.hindawi.com

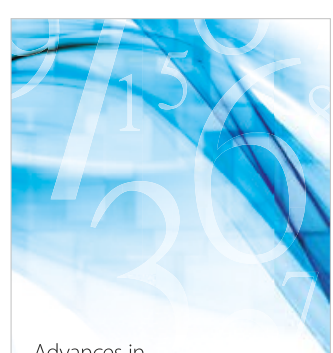

Advances in
Numerical Analysis
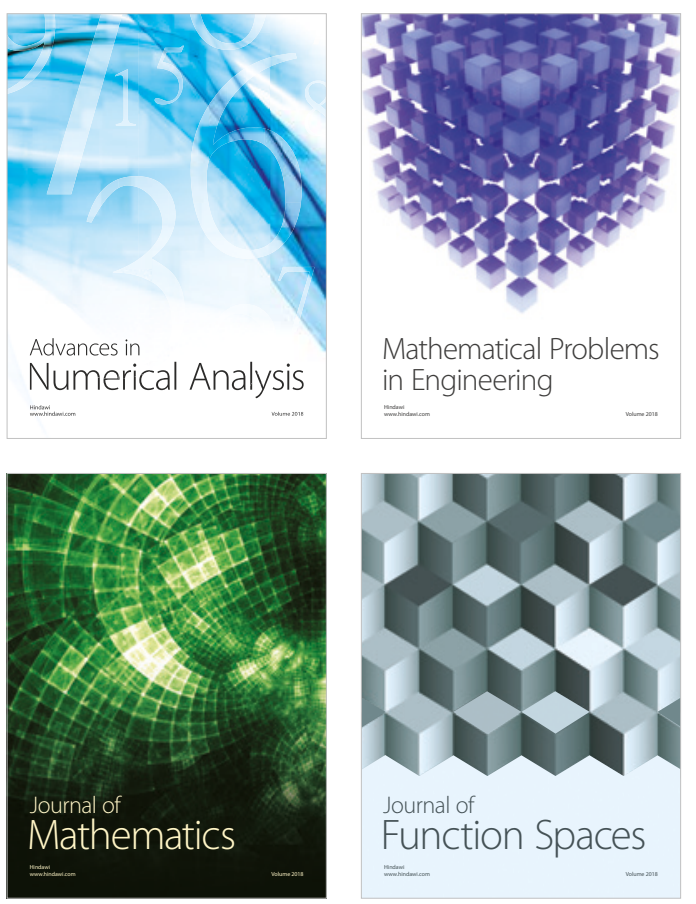

Mathematical Problems in Engineering

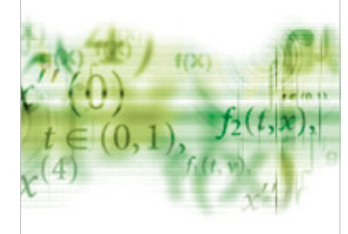

International Journal of

Differential Equations

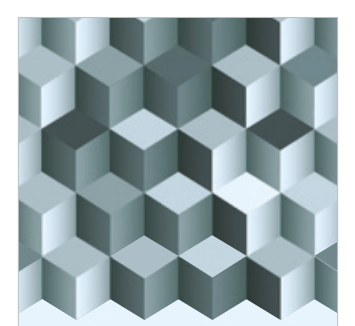

Journal of

Function Spaces

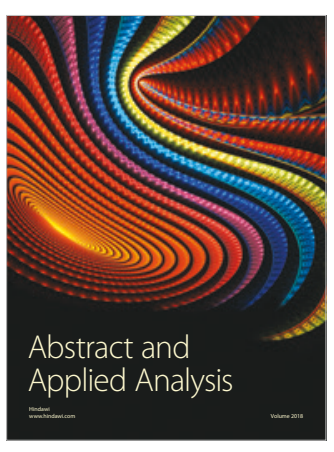

The Scientific

World Journal

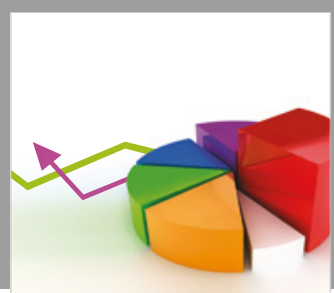

Journal of

Probability and Statistics
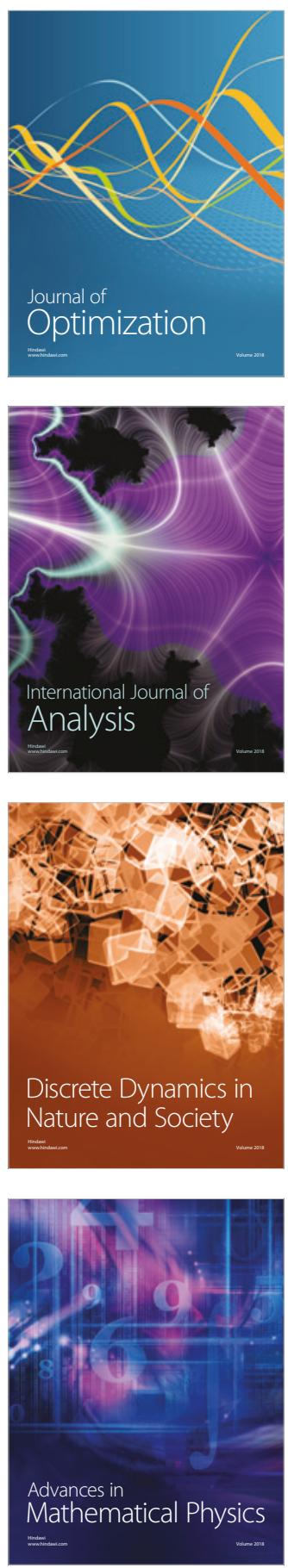